\title{
Resonant holographic interferometry measurements of laser ablation plumes in vacuum, gas, and plasma environments
}

\author{
R. A. Lindley, ${ }^{\text {a) }}$ R. M. Gilgenbach, ${ }^{\text {b) }}$ C. H. Ching, and J. S. Lash \\ Intense Energy Beam Interaction Laboratory, Nuclear Engineering Department, \\ University of Michigan, Ann Arbor, Michigan 48109-2104 \\ G. L. Doll \\ Physics Department, General Motors Research and Development Center, Warren, Michigan 48090-9055
}

(Received 31 January 1994; accepted for publication 22 July 1994)

\begin{abstract}
Resonant holographic interferometry and dye-laser-resonance-absorption photography have been utilized to investigate the expansion of the laser ablation plumes produced by a $\mathrm{KrF}$ excimer laser beam $(248 \mathrm{~nm})$ focused onto an aluminum target $\left(\approx 0.1 \mathrm{~cm}^{2}, 2-6 \mathrm{~J} / \mathrm{cm}^{2}\right)$. Plume expansion was studied in vacuum and in background argon gas pressures of $14 \mathrm{mTorr}, 52 \mathrm{mTorr}, 210 \mathrm{mTorr}, 1 \mathrm{Torr}$, and 35 Torr. The existing theory for the interpretation of resonant interferograms has been extended to account for Doppler shift effects, the diagnostic laser bandwidth, and the selective absorption of the laser beam. Absolute line densities in the range $4.3 \times 10^{13}-1.0 \times 10^{15} \mathrm{~cm}^{-2}$ have been measured in the ablation plumes, which imply measured Al neutral densities of up to $1 \times 10^{15} \mathrm{~cm}^{-3}$. The total number of Al neutral atoms in a plume has been measured to be $\approx 3 \times 10^{14}$, which corresponds to a surface etch rate of $\approx 1 \mathrm{~nm} /$ pulse. Expansion velocities in the range $1.1-1.4 \mathrm{~cm} / \mu$ s were measured for the pressures $\leqslant 210$ mTorr, while $\approx 0.3 \mathrm{~cm} / \mu$ s was measured for 1 Torr and $\approx 0.08 \mathrm{~cm} / \mu$ s was measured for 35 Torr. Ablation plume expansion into a 1 Torr rf argon plasma environment was compared with the expansion into a 1 Torr argon gas. The ablation plume appeared to expand and dissipate slightly faster in the plasma.
\end{abstract}

\section{INTRODUCTION}

Laser ablation has a number of important applications in industry and medicine. In industry it has the potential to micromachine integrated-circuit patterns (lithography), to drill holes on printed circuit boards, and for surface marking. Laser ablation deposition is also used in materials research to deposit thin films of semiconducting, conducting, and superconducting materials. In medicine, laser ablation is used for lithotripsy (kidney stones) as well as corneal and keratorefractive (eye) surgery. Finally, research is underway to develop laser ablation plumes as an $\mathrm{x}$-ray source for lithography as well as a lasing medium for $\mathrm{x}$-ray lasers.

Many different diagnostics have been used to investigate the expansion of laser ablation plumes. The first basic parameter of general interest for ablation plume expansion is the expansion velocity. The leading-edge expansion velocity has been measured using photographic techniques, such as shadowgraphy, ${ }^{1}$ absorption photography, ${ }^{2-6}$ emission photography, ${ }^{7}$ planar-laser-induced fluorescence (PLIF), ${ }^{8,9}$ schlieren photography, ${ }^{6,10}$ resonant schlieren photography, ${ }^{2-4,11}$ Michelson interferometry, ${ }^{12}$ and resonant holographic interferometry (RHI). ${ }^{11,13,14}$ The time-of-flight (TOF) expansion velocity has been measured using various TOF techniques, such as charged particle collection, ${ }^{15,16}$

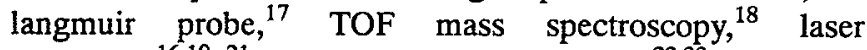
deflection, ${ }^{16,19-21}$ absorption spectroscopy, ${ }^{22,23}$ emission spectroscopy, ${ }^{16,23-25}$ laser-induced fluorescence, ${ }^{26-28}$ and TOF plasma recombination light (TOFPRL). ${ }^{29}$ In addition,

\footnotetext{
2)Present address: Physics Department, University of Sheffield, Sheffield S3 7RH, U.K.

b) Author to whom correspondence should be addressed.
}

the kinetic plume temperature has been inferred from the measured TOF expansion velocity. ${ }^{18,20,23,26,27,29}$

The second basic parameter of general interest in ablation plume expansion is the particle density. Langmuir probe $^{17}$ and laser deflection ${ }^{10,19}$ have been used to measure absolute electron densities, while Rayleigh scattering ${ }^{4}$ and $\mathrm{PLIF}^{8,9,28}$ have been used to measurc relative particle densities. Neutral particle density has also been measured through line-of-sight diagnostics. Absorption spectroscopy, ${ }^{22,29,30}$ Hook spectroscopy, ${ }^{31}$ Michelson interferometry, ${ }^{12}$ and $\mathrm{RHI}^{11,13,14}$ have been used to measure absolute particle line densities.

Two more parameters of interest in laser ablation are the particle yield and the ablation etch rate. The measurement of one of these parameters allows an estimation of the other. Total particle yields of ablation plumes have been measured using charged particle collection, ${ }^{15,16}$ and relative particle yields have been measured using TOF mass spectroscopy ${ }^{18,32}$ and laser deflection. ${ }^{16,19}$ The ablation etch rate has been measured using a mechanical stylus, ${ }^{33-35}$ scanning electron microscopy, ${ }^{35}$ and laser deflection. ${ }^{20}$

Previous work by the authors has utilized laser deflection, ${ }^{10,19-21}$ emission spectroscopy, ${ }^{24}$ Schlieren photography, ${ }^{6,10}$ dye-laser-resonance-absorption photography (DLRAP), ${ }^{5,6}$ and $\mathrm{RHI}^{14}$ to investigate laser ablation plume expansion. RHI has an advantage in that it is inherently an absolute line density diagnostic which is not sensitive to the magnitude of the detected light, a problem with LIF. Thus, RIII should be relatively immune to the effects of window coating in deposition experiments, compared to LIF and absorption spectroscopy. In this work, RHI and DLRAP have been utilized to investigate the two-dimensional temporal 
evolution of a single, resonant species (ground-state neutral aluminum) during laser ablation plume expansion. Here the RHI diagnostic was used to measure the leading edge expansion velocities, the absolute particle line densities, the total yield of the resonant species, and thus to cstimate the laser ablation etch rates under various background conditions.

\section{THEORY}

Holographic interferometry can be used to measure the phase shift of a laser beam incurred through a transparent medium. For nonresonant holographic interferometry, the laser bandwidth can be assumed to be infinitesimal and the interpretation of the phase shift is straightforward; however, for resonant interferometry, the incurred phase shift is a function of wavelength over the laser bandwidth. The observed fringe shift on the reconstructed interferogram can be calculated by averaging the electric fields of the phase-shifted photons incident on the holographic plate.

The electric field of the laser beam can be written

$\mathbf{E}\left(x, y, z, \omega, t_{L}\right)=S\left(x, y, t_{L}\right) \exp [i \omega t-i \Phi(x, y, z, \omega)]$,

where $(x, y)$ are the spatial coordinates on the holographic plate, $(z)$ is the direction of propagation of the laser beam, the term $S\left(x, y, t_{L}\right)$ includes the temporal and spatial pulse shape of the laser, and [dropping the $(x, y)$ notation]

$$
\begin{aligned}
\Phi(\omega, z) & =\frac{\omega}{c} \int_{0}^{z} \tilde{n}\left(\omega, z^{\prime}\right) d z^{\prime} \\
& =\frac{\omega z}{c}+\frac{\omega}{c} \int_{0}^{z}\left[\tilde{n}\left(\omega, z^{\prime}\right)-1\right] d z^{\prime} \\
& =\frac{\omega z}{c}+\phi(\omega, z)-i K(\omega, z),
\end{aligned}
$$

where $\tilde{n}\left(\omega, z^{\prime}\right)$ is the complex index of refraction, $z^{\prime}=0$ is the incident side of the beam splitter, and $\phi(\omega, z)$ and $K$ $(\omega, z)$ are real. For nonresonant holographic interferometry or for resonant holographic interferometry assuming an infinitesimal laser bandwidth, the observed resonant phase shift is simply determined using the equation

$$
\left(\frac{\Delta s}{s}\right)=\frac{\Delta \phi}{2 \pi}=\frac{\omega_{0}}{2 \pi c} \int_{0}^{z} \operatorname{Re}\left[\tilde{n}\left(\omega, z^{\prime}\right)-1\right] d z^{\prime} .
$$

If both a resonant and nonresonant phase shift are observed, the contributions due to neutrals, electrons, and resonant interaction can be recovered by taking three holographic interferograms at three different wavelengths, one of them very near resonance.

For wavelengths very close to a resonant atomic or molecular transition of a component gas species, the observed resonant phase shift is written as ${ }^{13,36}$

$$
\left(\frac{\Delta s}{s}\right)=\frac{\overline{N_{i} L} e^{2} f_{0}}{8 \pi m c \epsilon_{0}}\left(\frac{\omega_{0}-\omega}{\left(\omega-\omega_{0}\right)^{2}+\frac{\gamma^{2}}{4}}\right),
$$

where $\overline{N_{i} L}$ is the line integrated density of the resonant particles, $f_{0}$ is the oscillator strength of the transition with reso- nant frequency $\omega_{0}$, and $\gamma$ is the dissipative damping constant, the sum of the natural, Lorentz, Holtzmark, and Stark broadenings. Note that the natural broadening term is the sum of all of the Einstein coefficients for all of the possible relaxation transitions from both the upper state and the lower state of the particular transition in question. ${ }^{37}$ Also, Eq. (4) assumes $\left|\omega-\omega_{0}\right|$ is at least several times larger than $\gamma / 2$ and that Doppler broadening effects can be ignored.

When $\left|\omega-\omega_{0}\right| \approx \gamma / 2$ and when Doppler broadening is significant in comparison to other line-broadening mechanisms, the observed resonant phase shift as derived by Measures is ${ }^{38}$

$$
\left(\frac{\Delta s}{s}\right)=\frac{1}{2 \pi} \operatorname{Im}\left[-A \overline{N_{i} L} W(-u+i \alpha)\right],
$$

where

$$
\begin{aligned}
& W(z)=\left[\exp \left(-z^{2}\right)\right][1+\operatorname{erf}(i z)], \\
& \beta=\frac{\omega_{0}}{c}\left(\frac{2 k_{B} T}{m}\right)^{1 / 2}, \quad \alpha=\frac{\gamma_{i k}}{2 \beta}, \\
& u=\frac{\omega-\omega_{0}}{\beta}, \quad A=\left(\frac{\pi^{3 / 2} r_{0} c f_{i k}}{\beta}\right),
\end{aligned}
$$

and

$$
r_{0}=e^{2} /\left(4 \pi \epsilon_{0} m c^{2}\right)=2.818 \times 10^{-13} \mathrm{~cm}
$$

is the classical electron radius.

Dreiden et al. ${ }^{13}$ accounted for finite laser bandwidth by ignoring Doppler broadening [and thus using Eq. (4)], assuming that the line integrated density of the test section was near the lower detectability limit (i.e., ignoring selective absorption), and by doing a simple average of the calculated fringe shift over the full width at half-maximum (FWHM) of the laser line,

$$
\begin{aligned}
\overline{\left(\frac{\Delta s}{s}\right)} & =\frac{\overline{N_{i} L} r_{0} c f_{0}}{2 \delta \omega} \int_{\omega-(\delta \omega / 2)}^{\omega+(\delta \omega / 2)} d \omega_{1} \frac{\omega_{0}-\omega_{1}}{\left(\omega_{1}-\omega_{0}\right)^{2}+(\gamma / 2)^{2}} \\
& =\frac{\overline{N_{i} L} r_{0} c f_{0}}{2 \delta \omega} \frac{1}{2} \ln \left(\frac{1+x}{1-x}\right)=\frac{\overline{N_{i} L} r_{0} c f_{0}}{2 \delta \omega} \operatorname{arctanh}(x),
\end{aligned}
$$

where

$$
x=\frac{\left(\omega_{0}-\omega_{1}\right) \delta \omega}{\left(\omega_{1}-\omega_{0}\right)^{2}+(\gamma / 2)^{2}+(\delta \omega / 2)^{2}},
$$

and $\delta \omega$ is the FWHM bandwidth of the laser intensity.

The ablation plumes in this work are assumed to have a Maxwellian parallel velocity distribution (i.e., as seen by the dye laser beam) in the range $0.1-1.0 \mathrm{eV}^{26}$ and the dye laser used in this work has a laser bandwidth of $\approx 0.0031 \mathrm{~nm}$. Aluminum targets were ablated and the $\mathrm{Al}(\mathrm{I}), 394.401 \mathrm{~nm}$, resonance line $\left(f_{0}=0.115\right)$ was probed. Figure 1 compares the fringe shift interpretation curves of $\mathrm{Griem}^{36}$ [Eq. (4)], Measures $^{38}$ [Eq. (5)], and Dreiden et al. ${ }^{13}$ [Eq. (8)] for parameters relevant to this experiment. Griem's interpretation assumes a standard, cold, gaseous medium and an infinitesimal laser bandwidth. Notice that for either an increase in laser bandwidth (Dreiden et al.) or an increase in temperature (Measures), the maximum fringe shift per unit line density $(\Delta s / s) / N L$ drops dramatically and occurs at a larger $\Delta \lambda$. 


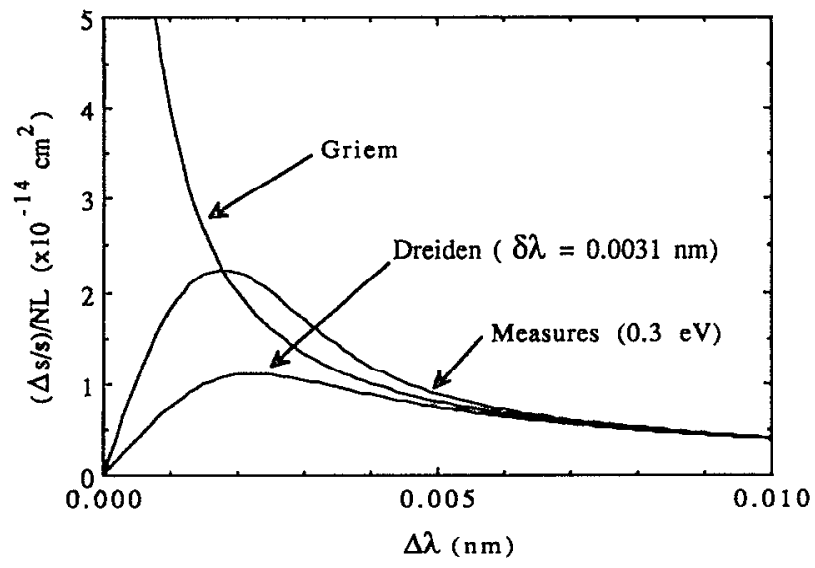

FIG. 1. Theoretical comparison of fringe shift interpretation curves for the $\mathrm{Al}(\mathrm{I}), 394.401 \mathrm{~nm}$ resonant transition, with $f_{0}=0.115$ and assuming $\gamma=1.47 \times 10^{8} \mathrm{~Hz}$. Griem's theory assumes a cold plume and an infinitesimal dye laser bandwidth. This curve is shaped like the other two, with the peak at the point $\left(6.06 \times 10^{-6} \mathrm{~nm}, 3.31 \times 10^{-12} \mathrm{~cm}^{2}\right)$. Dreiden and co-workers' theory averages the fringe shift over the full width at half-maximum (FWHM) of the dye laser bandwidth for a cold plume, and Measures's theory averages the fringe shift over a warm, Maxwellian distribution of resonant particles for an infinitesimal dye laser bandwidth.

Figure 2 shows how Measures's theory varies with temperature and compares it with the Gaussian laser bandwidth of our experiment. First note that as the temperature decreases the interpretation curve tends to return to Griem's interpretation curve. Second, note that when the dye laser has a finite bandwidth and is tuned to a small $\Delta \lambda$, such as 0.003 $\mathrm{nm}$, the value of $(\Delta s / s) / N L$ varies significantly over the laser bandwidth.

Whereas line density $N L$ versus the fringe shift $\Delta s / s$ is a linear relationship at each $\Delta \lambda$ in Figs. 1 and 2, this is not the case for the theory developed here. The Appendix contains a new derivation for the fringe shift interpretation, extending Measures's theory to take into account the effect of the finite laser bandwidth. The result [Eq. (A21)] is

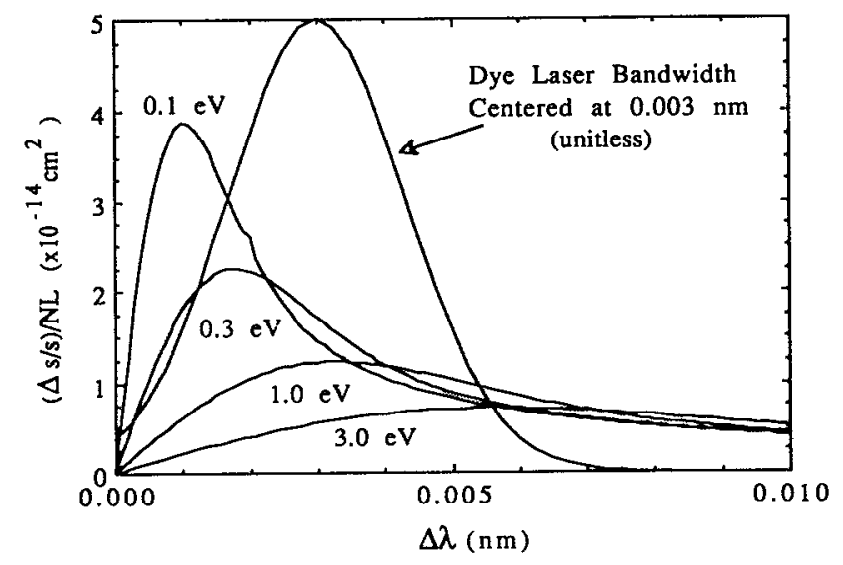

FIG. 2. Theoretical comparison of Measures's fringe shift interpretation curves for different plume temperatures, which assume an infinitesimal dye laser bandwidth. Also shown is an overlay of the Gaussian shape of the dye laser bandwidth, centered at $\Delta \lambda=0.003 \mathrm{~nm}$.
TABLE I. Example parameter values.

\begin{tabular}{l}
\hline Input: \\
Al(I), 394.401 nm \\
$f_{i k}=0.115$ \\
$\gamma_{i k}=1.47 \times 10^{8} \mathrm{~Hz}$ \\
$\Delta \lambda=0.003 \mathrm{~nm}$ \\
$T=0.3 \mathrm{eV}$ \\
$\sigma=1.60 \times 10^{10} \mathrm{rad} / \mathrm{s}$ \\
$(\delta \lambda=0.0031 \mathrm{~nm})$ \\
$N_{i} L=4 \times 10^{14} \mathrm{~cm}^{-2}$ \\
Output: \\
$\Delta=1.554$ \\
$\alpha=0.00314$ \\
$\beta=2.34 \times 10^{10} \mathrm{rad} / \mathrm{s}$ \\
$A=2.314 \times 10^{-13} \mathrm{~cm}^{-2}$ \\
$\epsilon=0.711-0.658 I$ \\
$\mathrm{FS}=4.43$ \\
\hline \hline
\end{tabular}

$$
\left(\frac{\Delta s}{s}\right)=\frac{1}{2 \pi} \operatorname{Im}\left[-4 \ln 2\left(\frac{\beta}{\sigma}\right)^{2} \epsilon_{s}^{2}-A \overline{N_{i} L} W\left(-\Delta-\epsilon_{\mathrm{s}}+i \alpha\right)\right]
$$

where $\sigma$ is the FWHM of the frequency of the laser intensity, $\Delta=\left(\bar{\omega}-\omega_{0}\right) / \beta$, and $\epsilon_{\mathrm{s}}$ is the numerically obtained root of the derivative of Eq. (A12). Table I shows example values of the parameters used in this work, and Fig. 3 shows a sample interferogram interpretation graph at $|\Delta \lambda|=0.003 \mathrm{~nm}$ as calculated by Griem ${ }^{36}\left[\mathrm{Eq}\right.$. (4)], Measures ${ }^{38}$ [Eq. (5)], Dreiden et $a .^{13}$ [Eq. (8)], and this work [Eq. (10)]. The first three are linear relationships with the slopes taken from Fig. 1. Notice that the value of Eq. (10) is the same as Measures's value for lower fringe shifts, while it increases to $77 \%$ larger than Measures's value at 8 fringe shifts. For $|\Delta \lambda|=0.010 \mathrm{~nm}$, though, the maximum difference in the four interpretation values at eight fringe shifts is less than $3 \%$, making any of the equations valid for $0.3 \mathrm{eV}$.

Since Fig. 2 shows that the fringe shift interpretation depends on the temperature chosen of the ablation plume, Fig. 4(a) shows Measures's interpretation at $\Delta \lambda=0.003 \mathrm{~nm}$ for the four curves shown in Fig. 2. Figure 4(b) shows the corresponding interpretation curves for this work. It shows that the interferogram interpretation is relatively insensitive

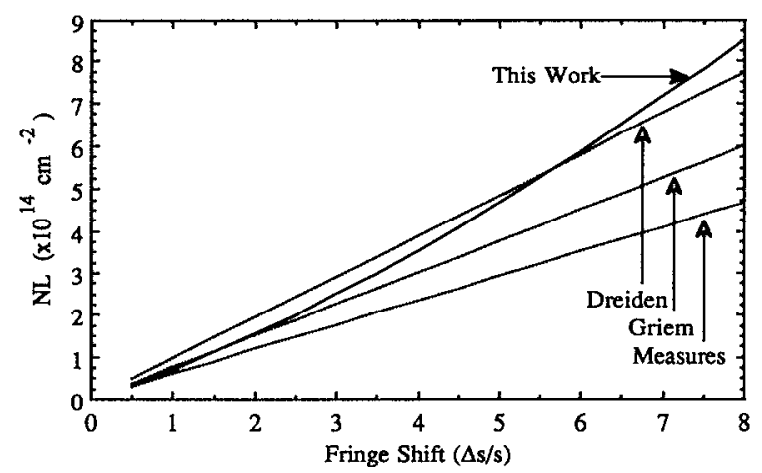

FIG. 3. Theoretical comparison of fringe shift interpretation curves at $\Delta \lambda$ $=0.003 \mathrm{~nm}$ assuming $T=0.3 \mathrm{cV}$. Notc that the slopes for the straight lines for Griem, Dreiden and co-workers, and Measures are taken from Fig. 1. 

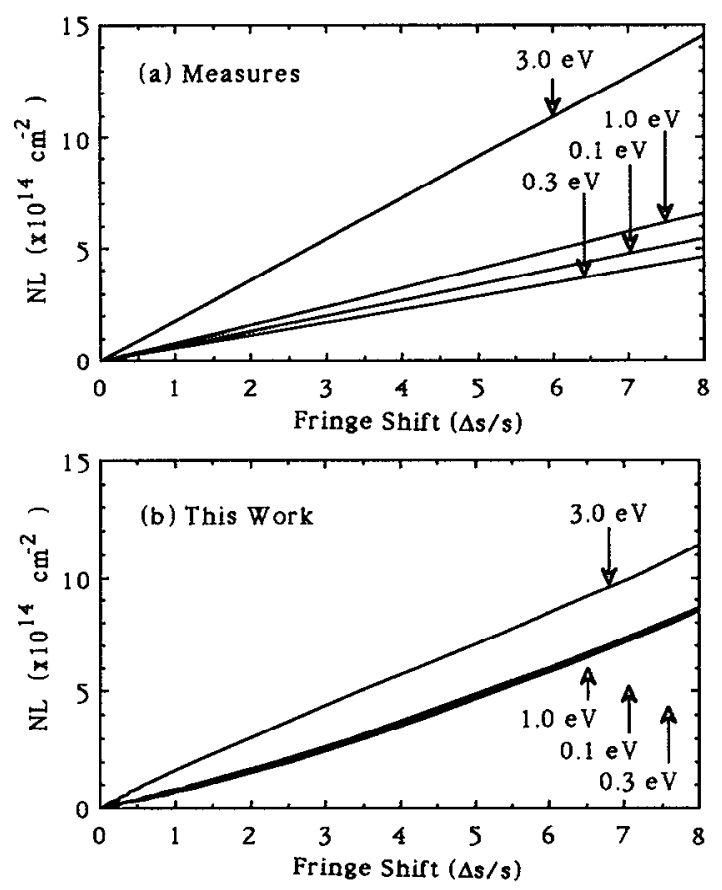

FIG. 4. Theoretical comparison of fringe shift interpretation curves at $\Delta \lambda$ $=0.003 \mathrm{~nm}$ as a function of plume temperature for (a) Measures's theory and (b) this work.

to the plume temperature for temperatures in the range $0.1-$ $1.0 \mathrm{eV}$. Since this is the parallel-particle, Maxwellian temperature range (i.e., parallel to the target surface and thus as seen by the laser beam) assumed for the plume in this work ${ }^{26}$ the line density results in this work are considered relatively insensitive to small changes in temperature from shot to shot. Again, as the $|\Delta \lambda|$ increases, the differences between the four theoretical curves decreases, so the theory presented in this work is only needed for very small values of $|\Delta \lambda|$.

\section{EXPERIMENTAL CONFIGURATION}

Figure 5 shows the basic experimental configuration for the optics table utilized for RHI and DLRAP. A KrF excimer laser $(248 \mathrm{~nm}, \leqslant 0.8 \mathrm{~J}, 40 \mathrm{~ns})$ was focused onto a solid aluminum target ( $98.5 \%$ pure) inside a glass, six-way, vacuum chamber, thus producing an ablation plume. A quartz flat (not shown) was used to split off part of the beam onto a calorimeter to monitor the shot-to-shot energy. To probe the ablation plume, a $\mathrm{XeCl}$ excimer-pumped dye laser $(\approx 5 \mathrm{~mJ}, 20$ $\mathrm{ns}$, QUI dye) was tuned near the $394.401 \mathrm{~nm}, \mathrm{Al}(\mathrm{I})$, resonant transition, expanded and collimated, and passed through the ablation plume.

To make an interferogram of the ablation plume, the dye laser beam was first split into two beams by an uncoated, quartz interferometry flat turned $70^{\circ}$ from normal incidence. Both beams were enlarged using a Galilean telescope and had an iris located at the focal point to remove uncollimated laser light. Mirrors were positioned and oriented to direct the collimated beams onto the holographic plate (Agfa-Gavert 8E56) and to make the difference in the beam path lengths less than the coherence length of the dye laser $(\approx 5 \mathrm{~cm})$. A blue glass filter with peak transmission at $400 \mathrm{~nm}$ was mounted on the entrance window of the holographic camera to keep out room light during the experiment.

The experimental procedure was as follows. First, only the dye laser was pulsed to expose the holographic plate with a without-the-plume interference pattern. Next, the rotatable mirror in the reference beam was turned very slightly to produce straight, background fringes on the reconstructions. Then the ablating laser and the dye laser were pulsed with a relative delay to form a second interference pattern on the holographic plate, this pattern containing the ablation plume information. Four such double-pulsed holographic interferograms were taken on each $10.2 \times 12.7 \mathrm{~cm}^{2}$ holographic plate before the holographic camera was reloaded. After development in a darkroom, the interferograms were reconstructed using a HeNe laser and Polaroid 667 film. Reconstruction with a laser wavelength other than the exposure wavelength does not change the image for a transmission interferogram, just the image location and magnification. ${ }^{39}$ The scale in each interferogram was determined by placing a transparent ruler near the ablation target and holographically recording the ruler next to the ablation plume.

Careful attention was given to the temperature of the dye laser grating during an experimental run since the calibration (the dye laser wavelength control unit readout versus the actual output wavelength) changes with temperature. The control unit was calibrated at the beginning of each run by taking four interferograms across the line center using the smallest wavelength increment available on the grating control unit $(0.001-0.002 \mathrm{~nm})$. The interferogram which displayed the most absorption and the change in fringe shift direction (with respect to the interferograms before and after it) was the line center, $394.401 \mathrm{~nm}$, and thus $\Delta \lambda=+0.000$ nm.

For DLRAP the holographic camera light-tight box was removed from the holographic camera holder and the object

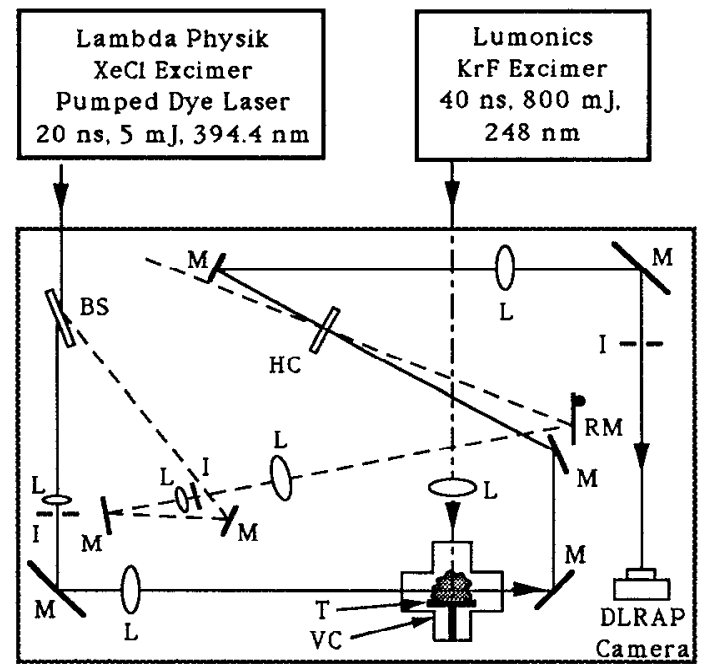

FIG. 5. Experimental configuration of the main optics table. I: iris; L: convex lens; M: mirror; RM: rotatable mirror; BS: beam splitter; $\mathrm{T}$ : aluminum target; VC: vacuum chamber; and HC: holographic camera. 
beam was passed through the blue glass plate, neutral density filters $(\mathrm{ND} \approx 3.0$, not shown), and into the DLRAP camera, which uses Polaroid type-47 film.

In order to observe the effects of a rf plasma on plume expansion, a steady-state ( $<10$ s to avoid oscillator overheating) If plasma was produced in the region of plume expansion. A $7 \mathrm{MHz} \approx 50 \mathrm{~W}$, rf generator was connected to a pair of $8.4-\mathrm{cm}$-diam brass parallel plates separated by $4.8 \mathrm{~cm}$ inside the vacuum chamber. The aluminum target face was positioned so that it was midway between and just slightly outside the parallel plates. This allowed the plume to expand between the parallel plates while not letting the target significantly perturb the electric field between the plates. The if voltage was turned on for about $3 \mathrm{~s}$ before the ablating laser was fired, thus allowing the if circuitry and the plasma sufficient time to equilibrate. The motivation for the rf plasma environment was to study possible changes in plume hydrodynamics and chemistry (currently under investigation).

\section{RESULTS AND DISCUSSION}

Figure 6 shows a temporal series of interferograms in vacuum ( $0.2 \mathrm{~m}$ Torr air). The ablation plume begins close to the surface and expands away from the target at later times. The reference fringes are shown vertically, and any deviation is fringe shift due to ground-state $\mathrm{Al}(\mathrm{I})$ atoms. This fringe shift is shown to the right-hand side as equifringe-shift contours, where each contour (from the outside to the inside) represents an increment of 0.5 fringe shift. It is assumed that the aluminum neutral atoms experience collisions, and that almost all of the electrons have recombined with the ions, at very early times $(\leqslant 0.2 \mu \mathrm{s})$. The peak aluminum neutral line density on each interferogram $\left(>5 \times 10^{14} \mathrm{~cm}^{-2}\right)$ is localed at the surface at early times $(0.29$ and $0.50 \mu \mathrm{s})$ and then separates from the surface at later times $(0.70,0.90$, and $1.11 \mu \mathrm{s})$. This corresponds to inferred particle densities of up to $1 \times 10^{15} \mathrm{~cm}^{-3}$. Also, the peak aluminum neutral line density decreases at later times to $\approx 1 \times 10^{14} \mathrm{~cm}^{-2}$ (at $1.11 \mu \mathrm{s}$ ). Notice the small shot-to-shot variations in the plume shape, although peak line densities were quite reproducible. It should be noted that the line density contour spacing is not constant; this is the main result of the theory derived in this work and thus each contour line must be referenced in the figure caption.

Figure 7(a) shows the total number of aluminum neutral atoms on the interferogram $N_{0}$, as a function of time for the vacuum time scan tracings presented in Fig. 6 . The parameter $N_{0}$ is determined by first tracing the contour plots onto fine graph paper and counting the number of squares in each region of fringe shift. This area is then multiplied by the calculated line density of the region to give the number of atoms, treating the plume as having step-function line densities. The sum of the number of atoms from each region is $N_{0}$. The value $N_{0}$ determined for the interferograms at 0.70 , 0.90 , and $1.11 \mu$ s is essentially the same, $3 \times 10^{13}$, and shows consistency with time even when the peak line density decreases from $2.5 \times 10^{14}$ to $1.4 \times 10^{14} \mathrm{~cm}^{-2}$.

Figure 7(b) plots the farthest extent of the $1 \times 10^{14} \mathrm{~cm}^{-2}$ equicontour line as a function of time for the interferograms in Fig. 6 . The expansion velocity $v$ was determined by calculating the best fit to a line passing through the first two

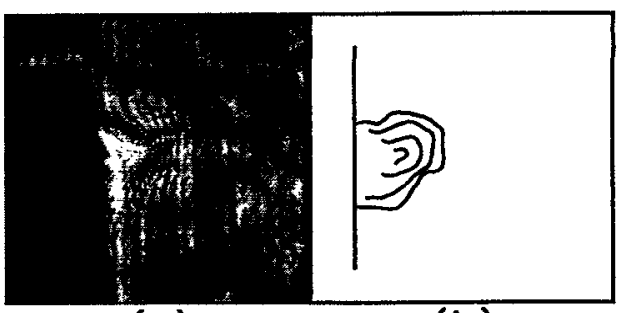

(a)

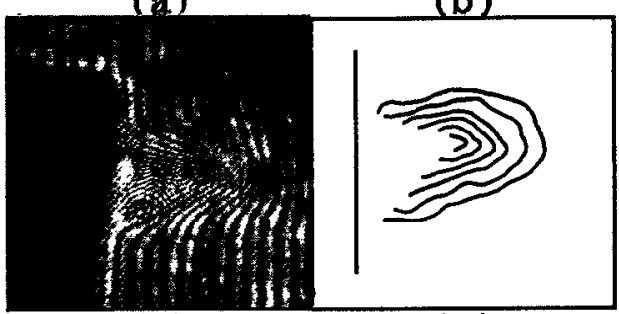

(c)

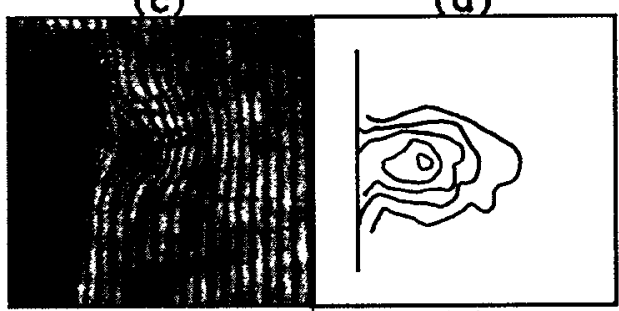

(e)
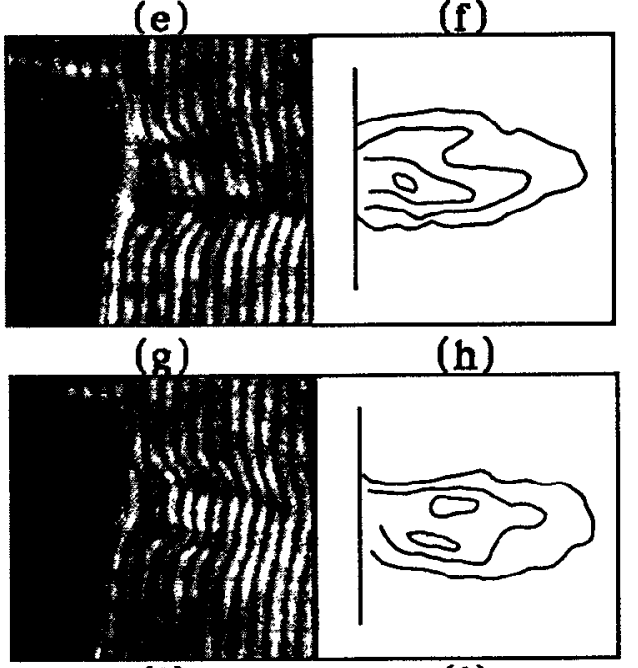

(i)

(j)

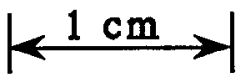

FIG. 6. Time scan in vacuum. Interferograms taken at $\Delta \lambda=+0.004 \mathrm{~nm}$, $\approx 2.4 \mathrm{~J} / \mathrm{cm}^{2}$ and their respective equicontour plots, where the lines correspond to increasing fringe shift. Interferogram (a) taken at $0.29 \mu \mathrm{s}$, and the lines from outer to inner in (b) represent $0.5 \mathrm{FS}=4.3 \times 10^{13} \mathrm{~cm}^{-2}$, $1.0 \mathrm{FS}=9.0 \times 10^{13} \mathrm{~cm}^{-2}, 2.0 \mathrm{FS}=1.9 \times 10^{14} \mathrm{~cm}^{-2}$, and $3.0 \mathrm{FS}=3.0 \times 10^{14}$ $\mathrm{cm}^{-2}$. Interferogram (c) taken at $0.50 \mu \mathrm{s}$, and the lines from outer to inner in (d) represent $0.5 \mathrm{FS}=4.3 \times 10^{13} \mathrm{~cm}^{-2}, 1.0 \mathrm{FS}=9.0 \times 10^{13} \mathrm{~cm}^{-2}$, $2.0 \mathrm{FS}=1.9 \times 10^{14} \mathrm{~cm}^{-2}, 3.0 \mathrm{FS}=3.0 \times 10^{14} \mathrm{~cm}^{-2}, 4.0 \mathrm{FS}=4.2 \times 10^{14} \mathrm{~cm}^{-2}$, and $5.0 \mathrm{FS}=5.5 \times 10^{14} \mathrm{~cm}^{-2}$. Interferogram (e) taken at $0.70 \mu \mathrm{s}$, and the lines from outer to inner in (f) represent. $0.5 \mathrm{FS}=4.3 \times 10^{13} \mathrm{~cm}^{-2}$, $1.0 \mathrm{FS}=9.0 \times 10^{13} \mathrm{~cm}^{-2}, 1.5 \mathrm{FS}=1.4 \times 10^{14} \mathrm{~cm}^{-2}, 2.0 \mathrm{FS}=1.9 \times 10^{14} \mathrm{~cm}^{-2}$, and $2.5 \mathrm{FS}=2.5 \times 10^{14} \mathrm{~cm}^{-2}$. Interferogram $(\mathrm{g})$ taken at $0.90 \mu \mathrm{s}$, and the lines from outer to inner in (h) represent $0.5 \mathrm{FS}=4.3 \times 10^{13} \mathrm{~cm}^{-2}$, $1.0 \mathrm{FS}=9.0 \times 10^{13} \mathrm{~cm}^{-2}, 1.5 \mathrm{FS}=1.4 \times 10^{14} \mathrm{~cm}^{-2}$, and $2.0 \mathrm{FS}=1.9 \times 10^{14}$ $\mathrm{cm}^{-2}$. Interferogram (i) taken at $1.11 \mu \mathrm{s}$, and the lines from outer to inner in (j) represent $0.5 \mathrm{FS}=4.3 \times 10^{13} \mathrm{~cm}^{-2}, 1.0 \mathrm{FS}=9.0 \times 10^{13} \mathrm{~cm}^{-2}$, and $1.5 \mathrm{FS}=1.4 \times 10^{14} \mathrm{~cm}^{-2}$. 

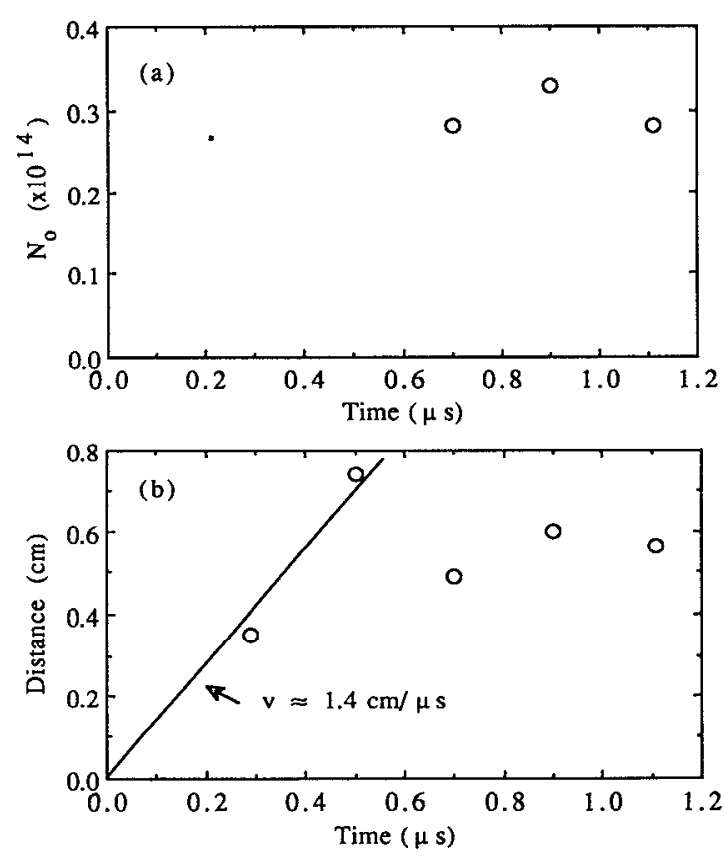

FIG. 7. Time scan in vacuum interpretation: (a) the total number of aluminum neutral atoms $N_{0}$ vs time, and (b) the expansion of the $1 \times 10^{14} \mathrm{~cm}^{-2}$ equicontour line vs time.

points as well as the origin. The expansion velocity of $\mathbf{1 . 4}$ $\mathrm{cm} / \mu \mathrm{s}$ is consistent with other recent measurements of the TOF, most probable expansion velocity, ${ }^{23,28}$ and the leading-edge expansion velocity ${ }^{6,12,26}$ of laser ablation expansion in vacuum.

Two series of interferograms were taken at $0.5 \mu \mathrm{s}$ at various wavelengths in order to determine the consistency of measuring the line density for an ablation plume over a range of dye laser wavelengths. The first set of interferograms used a fluence of $2.0-2.7 \mathrm{~J} / \mathrm{cm}^{2}$ and the second set used a fluence of $4.2-4.7 \mathrm{~J} / \mathrm{cm}^{2}$. Figure 8 shows the results as a function of wavelength for the two scans at a point $0.7 \mathrm{~cm}$ away from the target along the line of symmetry of the ablation plume. First, note that absorption prevented the measurement of

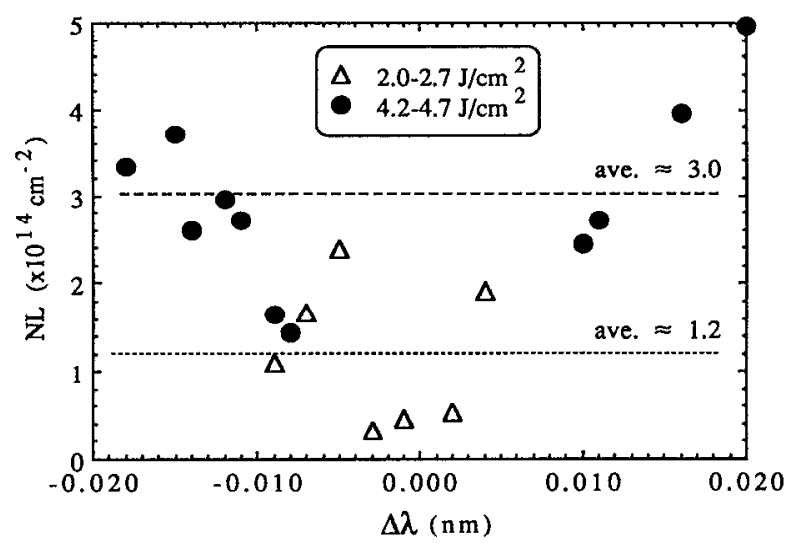

FIG. 8. Wavelength scan in vacuum. Line density vs wavelength at $0.5 \mu \mathrm{s}$ for two fluences to show the consistency of line density measurement as a function of wavelength. fringe shift at $0.7 \mathrm{~cm}$ on the interferogram for $|\Delta \lambda|<0.009$ $\mathrm{nm}$ for the higher-laser-fluence case. Second, note that the measured aluminum neutral line density seemingly increases with increasing $|\Delta \lambda|$. This is because the measured fringe shifts at this point on each of the interferograms were around 1.0 fringe shift and were measured to the nearest 0.25 fringe shift. Thus, Fig. 8 looks similar to a theoretical curve showing the aluminum neutral line density interpretation as a function of wavelength for a constant 1.0 fringe shift. Third, note that the ratio of the line density averages for the two fluences is $\approx 2.5$, which is comparable to, yet larger than, the ratio of the average laser fluences, $\approx 1.9$. It is suggested that if the fringe shifts at an identical point on a series of interferograms were measured with greater certainty (such as $5.00 \pm 0.25$ fringe shifts instead of $1.00 \pm 0.25$ fringe shifts), then the graph of the measured line density versus wavelength would be more constant as a function of wavelength for each fluence. It is also possible that the temperatures in the plumes at the two different fluence ranges could be significantly different, and thus affecting the outcome of Fig. 8. A temperature of $0.3 \mathrm{eV}$ was assumed for both fluence ranges since the individual plume temperatures could not be determined at that time.

Figures 9(a)-9(1) show another series of interferograms in vacuum, except this time for a laser ablative deposition configuration in which the laser is incident on the aluminum target with an angle of $\approx 70^{\circ}$ and an amorphous-siliconcoated silicon wafer has been placed $15 \mathrm{~mm}$ away from the aluminum target. The expanded dye laser beam was not perfectly parallel to the target and wafer surfaces for this experimental run, so the separation distance appears to be only $\approx 12.5 \mathrm{~mm}$ in the reconstructed interferograms.

The ablation laser energy was higher for this run, so the fluence is higher for the interferograms in Fig. 9 than for Fig. 6 , even though the target was tilted and thus the spot size was larger. The ablation plume begins close to the surface and expands toward the silicon wafer at earlier times $(0.25$, 0.52 , and $1.00 \mu \mathrm{s})$. By $1.21 \mu \mathrm{s}$ the ablation plume makes contact with the wafer and then by $1.69 \mu$ s the plume peak density is observed to begin receding back to the aluminum surface. Thus, while the aluminum neutrals are continually expanding outward, the RHI-detectable portion of the aluminum neutral atoms appears to extend from and then recede back to the aluminum target surface.

Notice that the outermost equiline-density contour in Fig. $9(j)$ is $1.1 \times 10^{14} \mathrm{~cm}^{-2}$, while the innermost line density in Fig. 9(1) is $6.9 \times 10^{13} \mathrm{~cm}^{-2}$, showing that the line densities represented in the former drawing have all nearly receded to the target surface. Also notice that the peak line density for each interferogram appears to remain at the target surface throughout the time scan. The maximum line density for the time scan, $\approx 6 \times 10^{14} \mathrm{~cm}^{-2}$, infers a maximum particle density of $\approx 5 \times 10^{14} \mathrm{~cm}^{-3}$.

Figures 10(a)-10(h) show a series of DLRAPs that correspond to the interferograms in Fig. 9. During the experimental run four interferograms were taken and then the corresponding four DLRAPs were taken under the same conditions. Here, also, the ablation plume maximum density is seen to expand toward the wafer at earlier times $(0.21 \mu \mathrm{s})$, 


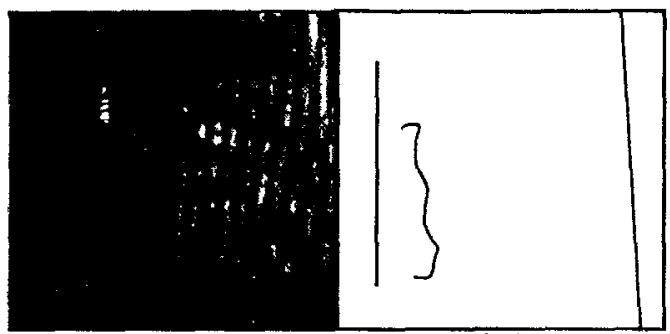

(a)

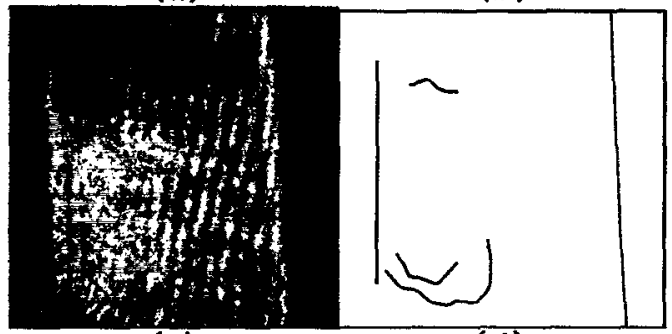

(c)

(d)

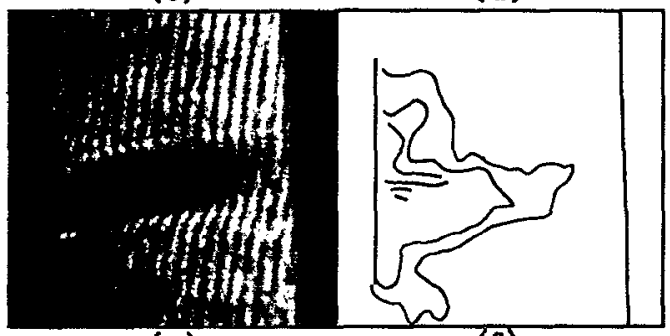

(e)

(f)

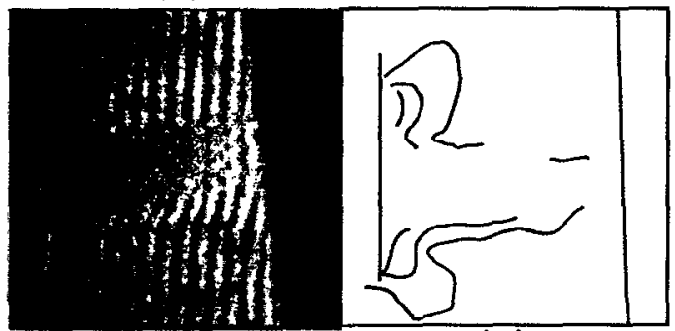

(8)

(h)

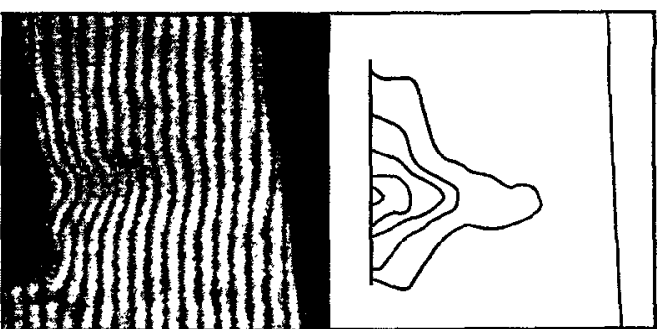

(i)

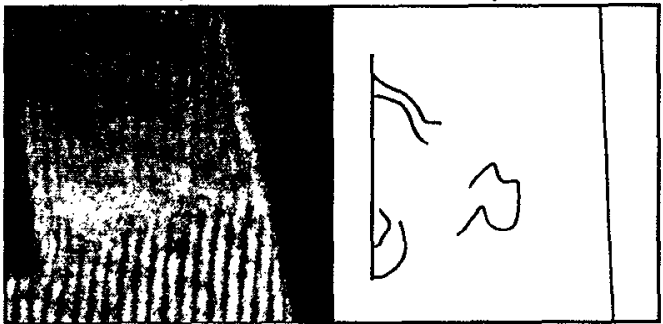

(k)

(1)

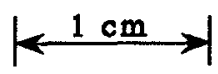

FIG. 9. Deposition time scan in vacuum. Interferograms and their respective equicontour plots, where the lines correspond to increasing fringe shift. An amorphous-silicon-coated silicon wafer at room temperature is mounted 15 mm away from the aluminum target and is on the right-hand side in the above interferograms. Interferogram (a) taken at $0.25 \mu \mathrm{s},-0.016 \mathrm{~nm}, \approx 5.4$ $\mathrm{J} / \mathrm{cm}^{2}$, and the line in (b) represents $0.5 \mathrm{FS}=2.0 \times 10^{14} \mathrm{~cm}^{-2}$. Interferogram (c) taken at $0.52 \mu \mathrm{s},-0.009 \mathrm{~nm}, \approx 6.2 \mathrm{~J} / \mathrm{cm}^{2}$, and the lines from outer to inner in (d) represent $0.5 \mathrm{FS}=1.1 \times 10^{14} \mathrm{~cm}^{-2}$, and $1.0 \mathrm{FS}=2.2 \times 10^{14} \mathrm{~cm}^{-2}$. Interferogram (e) taken at $1.01 \mu \mathrm{s},-0.009 \mathrm{~nm}, \approx 5.6 \mathrm{~J} / \mathrm{cm}^{2}$, and the lines from outer to inner in (f) represent $0.5 \mathrm{FS}=1.1 \times 10^{14} \mathrm{~cm}^{-2}$, $1.0 \mathrm{FS}=2.2 \times 10^{14} \mathrm{~cm}^{-2}, 1.5 \mathrm{FS}=3.3 \times 10^{14} \mathrm{~cm}^{-2}, 2.0 \mathrm{FS}=4.4 \times 10^{14} \mathrm{~cm}^{-2}$, $2.5 \mathrm{FS}=5.5 \times 10^{14} \mathrm{~cm}^{-2}$, and $3.0 \mathrm{FS}=6.6 \times 10^{14} \mathrm{~cm}^{-2}$. Interferogram $(\mathrm{g})$ taken at $1.21 \mu \mathrm{s},-0.016 \mathrm{~nm}, \approx 6.2 \mathrm{~J} / \mathrm{cm}^{2}$, and the lines from outer to inner in (h) represent $0.5 F S=2.0 \times 10^{14} \mathrm{~cm}^{-2}, 1.0 \mathrm{FS}=4.0 \times 10^{14} \mathrm{~cm}^{-2}$, and $1.5 \mathrm{FS}=5.9 \times 10^{14} \mathrm{~cm}^{-2}$. Interferogram (i) taken at $1.69 \mu \mathrm{s},-0.009 \mathrm{~nm}$, $\approx 6.2 \mathrm{~J} / \mathrm{cm}^{2}$, and the lines from outer to inner in (j) represent $0.5 F S=1.1 \times 10^{14} \mathrm{~cm}^{-2}, 1.0 \mathrm{FS}=2.2 \times 10^{14} \mathrm{~cm}^{-2}, 1.5 \mathrm{FS}=3.3 \times 10^{14} \mathrm{~cm}^{-2}$, $2.0 \mathrm{FS}=4.4 \times 10^{14} \mathrm{~cm}^{-2}$, and $2.5 \mathrm{FS}=5.5 \times 10^{14} \mathrm{~cm}^{-2}$. Interferogram (k) taken at $2.03 \mu \mathrm{s},-0.003 \mathrm{~nm}, \approx 6.2 \mathrm{~J} / \mathrm{cm}^{2}$, and the lines from outer to inner in (l) represent $0.5 \mathrm{FS}=3.2 \times 10^{13} \mathrm{~cm}^{-2}$ and $1.0 \mathrm{FS}=6.9 \times 10^{13} \mathrm{~cm}^{-2}$.

make contact with the wafer at intermediate times $(0.50$, $1.00,1.21$, and $1.61 \mu \mathrm{s})$, and recede from the wafer at later times $(2.00$ and $4.02 \mu \mathrm{s})$. By $5.02 \mu \mathrm{s}$ the ablation plume is no longer observable. Since the ablation plume was observed at $4.02 \mu \mathrm{s}$ with DLRAP but not with RHI, the former diagnostic is more sensitive.

The total number of aluminum neutral atoms at $1.69 \mu \mathrm{s}$ was $8.3 \times 10^{13}$; the parameter $N_{0}$ could only be determined for one interferogram [Fig. 9(i)] due to the absorption observed in this time scan. Since this interferogram, at $1.69 \mu \mathrm{s}$, was taken after the plume had impacted the deposition substrate and appeared to begin to recede back to the target surface, its measurement of $N_{0}$ is lower than the true number of aluminum neutral atoms leaving the target surface. When compared with the first time scan in vacuum, the plume expansion in Fig. 9 is much more directional, and thus a greater number of particles ( $>3$ times) expanding in a more forward direction produces absorption for a greater distance away from the target, even for larger $|\Delta \lambda|$. The variation in plume expansion shape has been observed to depend upon experimental parameters such as fluence magnitude, fluence uniformity, background pressure, and the distance from the laser focal lens to the target. ${ }^{9,14}$ An illustration of the measured variation in the expansion velocity is given in the last graph of the article (Fig. 18), while the variation in shot-toshot plume shape is evident in the interferograms throughout. Although there was shot-to-shot variation, the general plume shape (spherical, elliptical, bowl shaped, pine-tree shaped, etc.) was the same within an experimental run.

Concerning variation in the total number of particles counted, the hand integration of the interferograms results in an error of about $5 \%$ but the nature of the RHI diagnostic results in lower bound-particle counts. Particles outside the 0.5 fringe shift line are not counted while a constant line density of particles is assumed between each of the contours. Uncertainty in calculating the line density that a particular contour represents is mainly due to the uncertainty in determining the $\Delta \lambda$ for a given photograph. If the uncertainty in $\Delta \lambda$ is $+0.0005 \mathrm{~nm}$, then the uncertainty would be high for $0.000 \mathrm{~nm}<\Delta \lambda<0.005 \mathrm{~nm}$. However, for $\Delta \lambda>0.005 \mathrm{~nm}$, the 


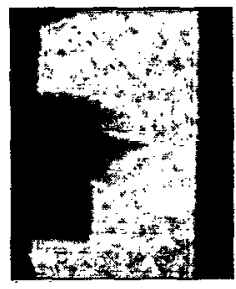

(a)

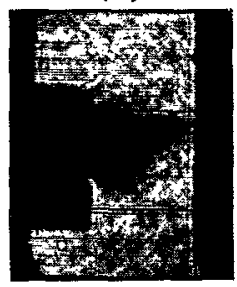

(b)

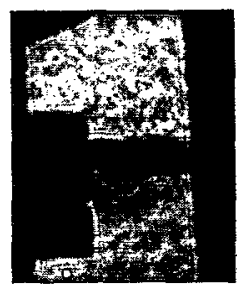

(c)

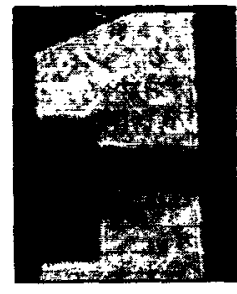

(d)

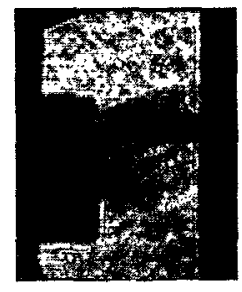

(e)

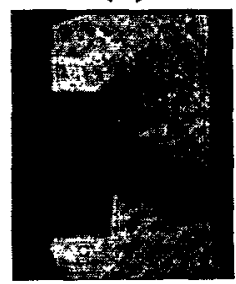

(f)

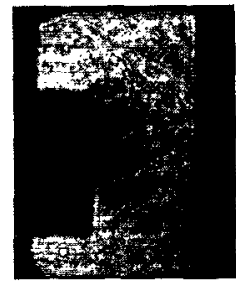

(g)

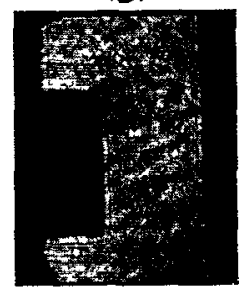

(h)

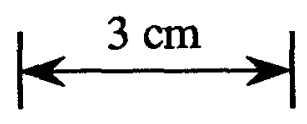

FIG. 10. Deposition time scan in vacuum. DLRAPs that correspond to, but are not simultaneous to, the interferograms in Fig. 9, plus two additional DLRAPs at later times: (a) $0.21 \mu \mathrm{s},-0.016 \mathrm{~nm}, \approx 5.4 \mathrm{~J} / \mathrm{cm}^{2}$; (b) $0.50 \mu \mathrm{s}$, $-0.009 \mathrm{~nm}, \approx 5.9 \mathrm{~J} / \mathrm{cm}^{2}$; (c) $1.00 \mu \mathrm{s},-0.009 \mathrm{~nm}, \approx 5.8 \mathrm{~J} / \mathrm{cm}^{2}$; (d) $1.21 \mu \mathrm{s}$, $-0.009 \mathrm{~nm}, \approx 5.4 \mathrm{~J} / \mathrm{cm}^{2}$; (e) $1.61 \mu \mathrm{s},-0.009 \mathrm{~nm}, \approx 5.4 \mathrm{~J} / \mathrm{cm}^{2}$; (f) $2.00 \mu \mathrm{s}$, $-0.003 \mathrm{~nm}, \approx 5.4 \mathrm{~J} / \mathrm{cm}^{2} ;(\mathrm{g}) 4.02 \mu \mathrm{s},-0.003 \mathrm{~nm}, \approx 5.8 \mathrm{~J} / \mathrm{cm}^{2} ;(\mathrm{h}) 5.02 \mu \mathrm{s}$, $-0.003 \mathrm{~nm}, \approx 4.8 \mathrm{~J} / \mathrm{cm}^{2}$. Note that the DLRAP image is inverted with respect to the interferograms of Fig. 9.

uncertainty is reasonably low. Figure 8 illustrates the uncertainty in determining the measured line density as a function of $\Delta \lambda$.

Figure 11 shows the expansion of the $1 \times 10^{14} \mathrm{~cm}^{-2}$ equicontour line as a function of time for the vacuum-deposition interferograms. The leading-edge expansion velocity of $\approx 1.1$ $\mathrm{cm} / \mu \mathrm{s}$ is about the same as the first-time scan in vacuum, $\approx 1.4 \mathrm{~cm} / \mu \mathrm{s}$. These expansion velocities can be used to calculate the initial temperature of the collisional ablation plume using the relation ${ }^{20}$

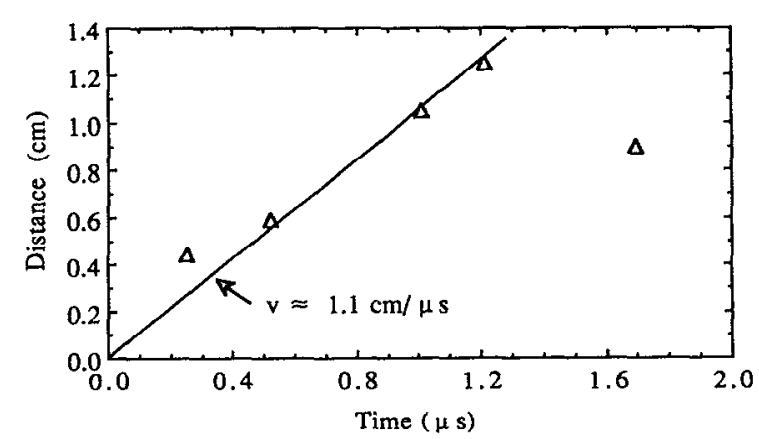

FIG. 11. Deposition time scan in vacuum interpretation; the expansion of the $1 \times 10^{14} \mathrm{~cm}^{-2}$ equicontour line vs time.

$$
k_{B} T=\frac{(y-1)^{2}}{2 \gamma} \frac{M u_{\max }^{2}}{2},
$$

where $y=1.667$ for an ideal, monatomic gas and $u_{\max }$ is the maximum expansion velocity measured for the laser ablation plume after the plume has become noncollisional. The velocities 1.1 and $1.4 \mathrm{~cm} / \mu$ s then relate to initial (perpendicular to target) plume temperatures of 2.3 and $3.6 \mathrm{eV}$, respectively. This falls within the temperature range measured by Dreyfus and co-workers ${ }^{26}$ using LIF on copper ablation for similar fluences: $\approx 0.26-\approx 4 \mathrm{eV}$.

Figure 12(a) shows a photograph of the amorphoussilicon-coated silicon wafer with the deposited aluminum

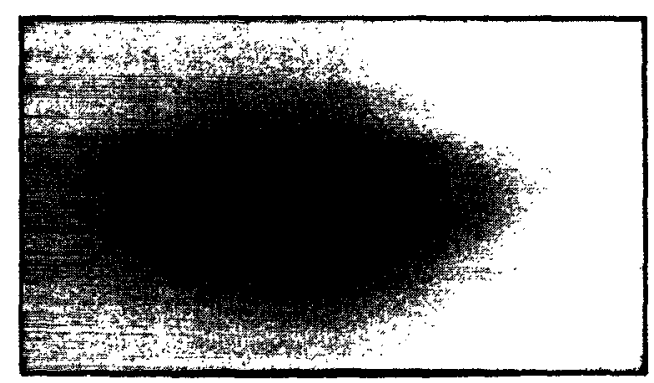

(a)

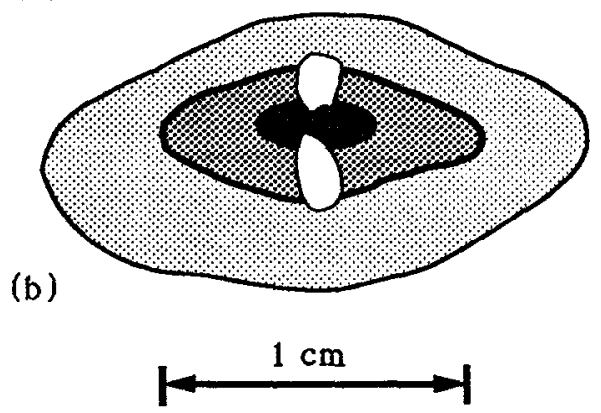

FIG. 12. (a) Laser ablation deposition substrate (amorphous-silicon-coated silicon wafer) after $55 \mathrm{KrF}$ ablation laser pulses $\left(\approx 4.5-6 \mathrm{~J} / \mathrm{cm}^{2}\right)$ on a $98.5 \%$ pure aluminum target. Target-substrate separation distance was $15 \mathrm{~mm}$; substrate was at room temperature. (b) A drawing of the Iaser ablation spot (white) overlaying the aluminum thin film deposited on the silicon substrate (shaded). Note that the direction of greater lateral plume expansion corresponds to the narrower dimension of the ablation spot. 
thin film after 55 ablation pulses, and Fig. 12(b) shows a rendition of the deposition spot (shaded) with an overlay of the laser ablation spot (white). The central region of the thin film was dark. The next region was somewhat lighter, and it was surrounded by a dark linc of material. The third region is an indication of the farthest extent of deposition, although little material was deposited there. Attempts to measure the aluminum thin-film thickness using ellipsometry and the four-point resistance probe method were unsatisfactory. Note the photographic orientations: up in Fig. 12 is down in Fig. 9 and up in Fig. 10. Also note that the width of the two central thin-film regions in Fig. 12(b) (inside the thick line, from top to bottom), as viewed by the dye laser (going left- to righthand side), are approximately the same width as the ablation plume in Fig. 9(e).

The double-ablation spot reflccts a poor laser intensity profile for the ablation excimer laser that was present for the interferograms and DLRAPs in Figs. 9 and 10. The laser output consisted of two rectangles of unequal energy when focused, and this intensity profile was exaggerated for laser ablative deposition because of the steep angle of incidence onto the target. Initial attempts to remedy this laser intensity profile, without overhauling the laser, have proven ineffective. The other interferograms presented in this work were taken using a good, nearly square laser intensity profile.

Figure 12(b) shows clearly that the ablation plume expanded differently in the $x$ and $y$ directions ( $z$ defined as perpendicular to the target and substrate surfaces), and that it expanded more quickly in the direction corresponding to the narrow width of the ablation spot. This sideways motion has been previously observed in atmospheric pressure air and modeled using hydrodynamic equations; ${ }^{40}$ i.e., the expansion of the ablation plume particles in the sideways direction is governed primarily by self-collision and thus self-diffusion mechanisms $\Gamma=-D \nabla n$. This is especially the case for expansion into vacuum and lower-pressure background gases.

For an initially $(<0.1 \mu \mathrm{s})$ uniform particle density, the more narrow dimension of the laser spot corresponds to a higher-density gradient and thus a higher expansion velocity. Thus, a circular ablation spot would produce a more nearly circular thin film (in vacuum and lower background pressures) while a rectangular ablation spot, similar to the case here, produces an oval thin film with the larger dimension turned $90^{\circ}$ with respect to the longer ablation spot dimension. ${ }^{40}$

Therefore, the shape of the thin film in Fig. 12 is an indication that aluminum-aluminum collisions play a significant role in the expansion of the ablation plumes studied here. It is also a confirmation of the previously utilized assumption that the ablation plume can be considered to be Maxwellian in distribution from the collisions at early times, and in quasiequilibrium over the duration of the dye laser pulse ( $20 \mathrm{~ns}$ ). This is not to say that a significant percentage of ablated particles are experiencing collisions at the same time the interferogram is taken (e.g., $0.2-2.0 \mu \mathrm{s}$ ); rather, the collisions the ablated particles experience at very early times, when the ablation plume has a high enough particle density, determine and " $f x$ " the velocity distribution that is observed at later times when collisions are no longer significant.
It has been calculated by NoorBatcha, Lucchese, and Zeiri, ${ }^{41}$ using a Monte Carlo simulation, that a quasiMaxwellian velocity distribution is obtained for a laser ablation plume if the average number of collisions per ablated particle is greater than $\approx 3$, which is based on the ablative removal of 0.8 monolayers per pulse for the specific case investigated. It has therefore been suggested by Kelly and Dreyfus $^{42}$ that it would be difficult to produce an ablation plume whose particles experience less than three collisions on average and thus not have a quasi-Maxwellian velocity distribution.

A zeroth-order estimate of the target ablation rate can be made using the measured spot size of $0.049 \mathrm{~cm}^{2}$ for the ablation spot on Fig. 12(b). The ablation rate of $3 \times 10^{14}$ aluminum atoms per pulse corresponds to a surface etching rate of $1 \mathrm{~nm} / \mathrm{pulse}$, which is close to the etching rate of 5-10 $\mathrm{nm} /$ pulse measured by Kelly and Rothenburg for $2.3 \mathrm{~J} / \mathrm{cm}^{2}$ ablation of aluminum. ${ }^{33}$ Since the interferogram represents only aluminum neutrals in the ground state, the measure of total ablated particles is probably low and thus partially accounts for the low ablation etch rate estimate.

A simple estimate of the amount of energy absorbed by the target per ablated particle can also be made. Since $\approx 300$ $\mathrm{mJ}$ reached the target surface (for Figs. 9 and 10) and with a tabulated reflectivity of $92.5 \%$ for aluminum, the target absorbed $\approx 22.5 \mathrm{~mJ}$ of energy to ablate $\approx 3 \times 10^{14}$ neutral atoms. This is $\approx 466 \mathrm{eV}$ absorbed by the target per ablated neutral atom. Since the kinetic energy of each atom was on the order of $\approx 1 \mathrm{eV}$, then almost all of the energy absorbed by the target remained in the target and was thermally dissipated. This agrees well with Dreyfus and co-workers, ${ }^{26}$ who stated that thermal ablation is the dominant ablation mechanism for metals.

Laser ablation plume expansion in vacuum has been observed and theoretically modeled by Utterback, Tang, and Friichtenicht, ${ }^{43}$ Talents, ${ }^{44}$ and Kools et al. ${ }^{45}$ to have the form of a sphere of expanding particles, with a Maxwellian distribution, whose center of mass is moving away from the target with a constant velocity. The interferograms in Figs. 6 and 9 do not appear to reflect this since the peak line density moves away from the target and then moves back toward the target at later times. The data appear to support the two-component theory of laser ablation expansion. One group of particles (ions accelerated by the ambipolar potential and resulting charge-exchange neutrals) energetically moves away from the target and undergoes self-similar expansion while the other group of particles stays very close (within a few $\mathrm{mm}$ ) to the target surface. Hereafter, these will be called the expanding plume and the residual plume for simplicity.

The expanding plume and the residual plume have been observed and discussed for laser ablation in vacuum by Geohegan using emission spectroscopy ${ }^{7}$ and by Bakos and coworkers using TOFPRL. ${ }^{30}$ The phenomenon is also visible in the PLIF data presented by Cappelli and co-workers; ${ }^{8}$ however, this should not be confused with discussions distinguishing between species within the expanding plume such as that of Saenger, ${ }^{46}$ who discussed the differential expansion of the four different kinds of atoms in the ablation of $\mathrm{YBa}_{2} \mathrm{Cu}_{3} \mathrm{O}_{7-x}$, or Kools et al., ${ }^{45}$ who observed a $\mathrm{CuCl}$ ex- 
panding plume and a slower $\mathrm{Cu}_{3} \mathrm{Cl}_{3}$ expanding plume following the ablation of copper in the presence of chlorine gas.

Therefore, the interferograms in Figs. 6 and 9 represent the RHI-detectable portion of the sum of the expanding plume and the residual plume. The separation for the two components was observed in one interferogram at $14 \mathrm{mTorr}$ and another at 52 mTorr. In most cases, however, the expanding plume was not detected after separation from the residual plume. It is important to clarify at this point that the perpendicular expansion velocities $v_{z}$ were larger than the parallel expansion velocities $v_{x}, v_{y}$, as seen in the interferograms, and that even the two parallel expansion velocities $v_{x}, v_{y}$ were different from each other, as seen by the shape of the deposited thin film. Since the dye laser passed parallel to the target surface, it is the ensemble, parallel expansion velocity distribution in the direction of the dye laser beam that is appropriate for use in the fringe shift interpretation calculations.

Dreyfus and co-workers measured the perpendicular expansion temperature (corresponding to $v_{z}$ ) to be in the range $0.26-4.0 \mathrm{eV}^{26}$ The perpendicular expansion temperature range estimated here, $2.3-3.6 \mathrm{eV}$, is consistent with Dreyfus and co-workers' measurements, and the parallel expansion temperature for the ablation plumes in this work is presumed to be lower, in the range $0.1-1.0 \mathrm{eV}$. Since the plume temperature was not measured directly for the ablation plumes in

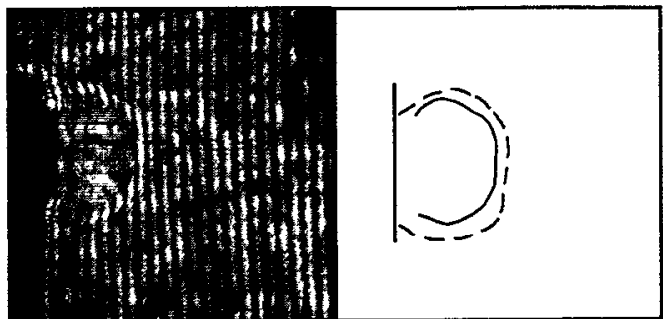

(a)

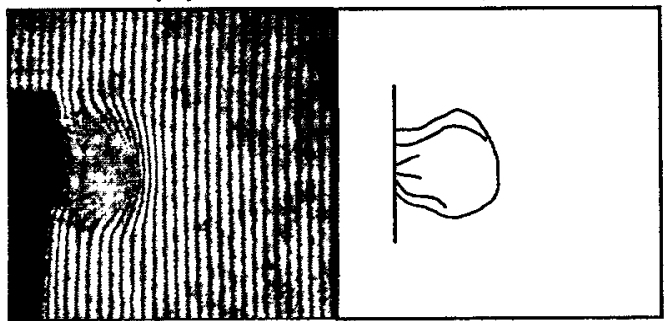

(c)

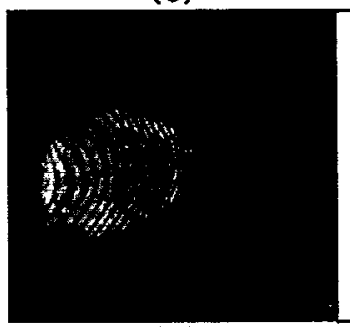

(e)

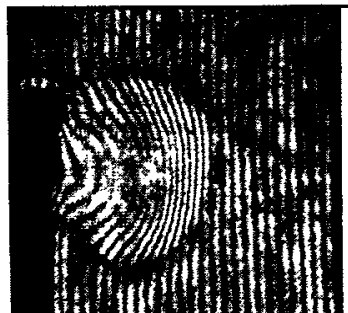

(

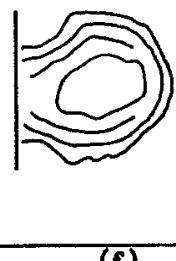

(f)

(d)

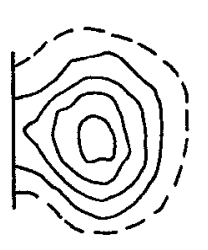

(b)

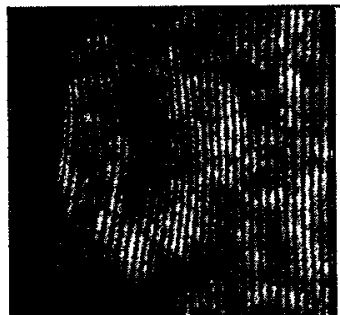

(i)

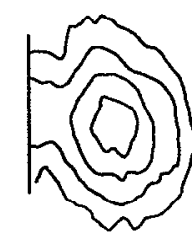

(j)

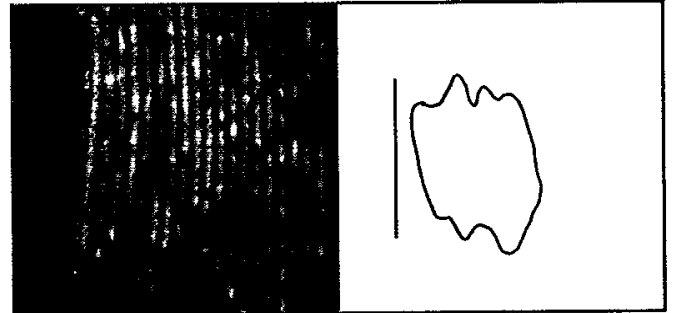

(k)

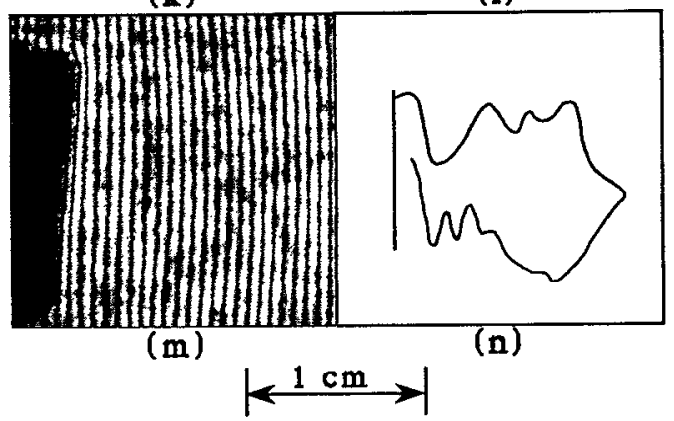

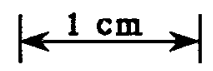

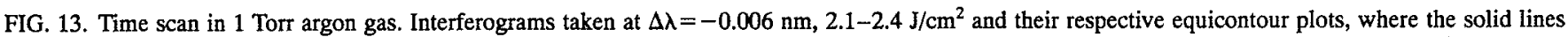

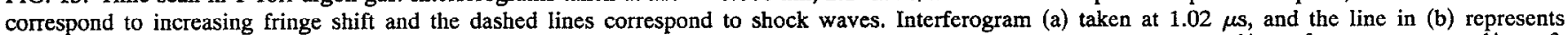

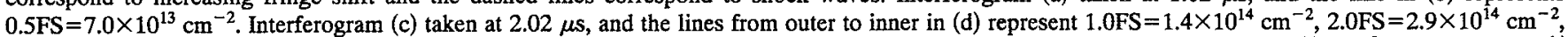

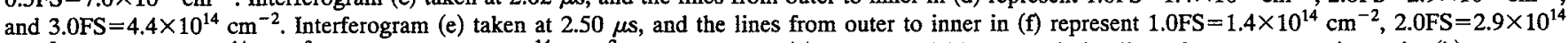

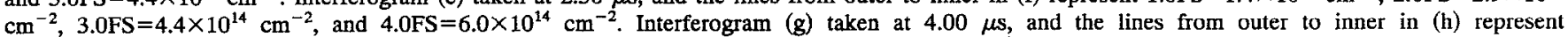

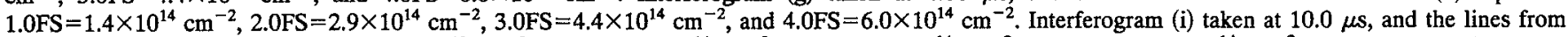

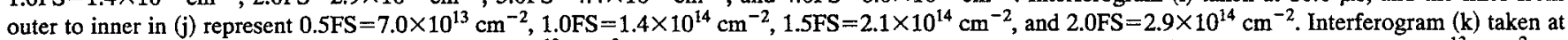
$20.0 \mu \mathrm{s}$, and the line in (1) represents $0.5 \mathrm{FS}=7.0 \times 10^{13} \mathrm{~cm}^{-2}$. Interferogram $(\mathrm{m})$ taken at $50.0 \mu \mathrm{s}$, and the line in (n) represents $0.5 \mathrm{FS}=7.0 \times 10^{13} \mathrm{~cm}-2$. 
this work, Dreyfus and co-workers' lower value, $0.3 \mathrm{eV}$, was chosen as the value to use in the fringe shift interpretation calculations. Again, it was shown above that the resonant interferogram interpretation calculation is insensitive to temperature for temperatures within the range $0.1-1.0 \mathrm{eV}$, so the absolute line densities, the expansion velocities for a given equiline-density contour, the total ablated number of atoms, and the ablation etch rate are all relatively insensitive to small temperature variations in this work.

Figure 13 shows a RHI time scan in 1 Torr argon gas. The first point to notice is that with a high enough background pressure a shock wave was observed at 1.02 and 4.00 $\mu \mathrm{s}$. Previously, DLRAP studies had only detected shock waves down to pressures of $\approx 5$ Torr. $^{6}$ It is presumed that a shock wave was present at the other times, but it was not detected as clearly as in Figs. 13(a) and 13(g). Second, notice that the plume expansion has slowed considerably with respect to the previous time scans at lower pressures.

Third, note the mushroom shape of the ablation plume. Sappey and Gamble ${ }^{9}$ attribute this shape for copper ablation plumes to the exothermic condensation of the atoms to dimers and to the fluid dynamics of one directionally expanding fluid in another. ${ }^{47}$ Fourth, notice that the plume appears to separate from the target at $20 \mu$ s. Finally, notice that the plume is also observed as late as $50.0 \mu$ s with RHI at 1 Torr; the latest time an ablation plume was observed in the previous vacuum data was $4.0 \mu \mathrm{s}$ in a DLRAP photo, which is more sensitive than RHI. Not only does this indicate that collisions with the argon gas are holding back the expanding ablation plume, but also that the conditions inside the plume are such that condensation of the aluminum neutral atoms is not significant. Kelly has recently proposcd a theory which may explain some features of this data. ${ }^{48}$

As with the holographic time scans at lower pressures, the peak line density in the earliest interferograms was obscured by absorption. The peak line density at 2.50 and 4.00 $\mu$ s was $6.0 \times 10^{14} \mathrm{~cm}^{-2}$, and it decreased to the detectability limit at this $|\Delta \lambda|, 7 \times 10^{13} \mathrm{~cm}^{-2}$, by $20.0 \mu \mathrm{s}$. These correspond to approximate aluminum neutral atom densities of $\approx 7 \times 10^{14}$ and $7 \times 10^{13} \mathrm{~cm}^{-3}$ for the earlier and later times, respectively.

Figure 14(a) shows the total number of aluminum neutral atoms counted for each interferogram versus time. The value of $N_{0}, \approx 3 \times 10^{13}-\approx 3 \times 10^{14}$, is similar to the values counted for the other time scans and the value clearly decreased with time as the aluminum atoms expand outward beyond the range of detection using RHI. Figure 14(b) shows the expansion of the $1 \times 10^{14} \mathrm{~cm}^{-2}$ equicontour as a function of time. The expansion velocity of $0.36 \mathrm{~cm} / \mu \mathrm{s}$ is nearly a third of the expansion velocity for the vacuum time scans, $1.1-1.4 \mathrm{~cm} / \mu \mathrm{s}$. The expansion of the $1 \times 10^{14} \mathrm{~cm}^{-2}$ equicontour then appeared to more or less stabilize at about $1 \mathrm{~cm}$ between 5 and $50 \mu \mathrm{s}$.

Figure 15 shows a RHI time scan in 1 Torr argon rf plasma. The rf plasma was produced between two parallel plates enclosing the region of plume expansion, as described above, and the with-rf interferograms (Fig. 15) were taken alternately from the without-rf interferograms (Fig. 13). The argon plasma is assumed to have a maximum electron den-
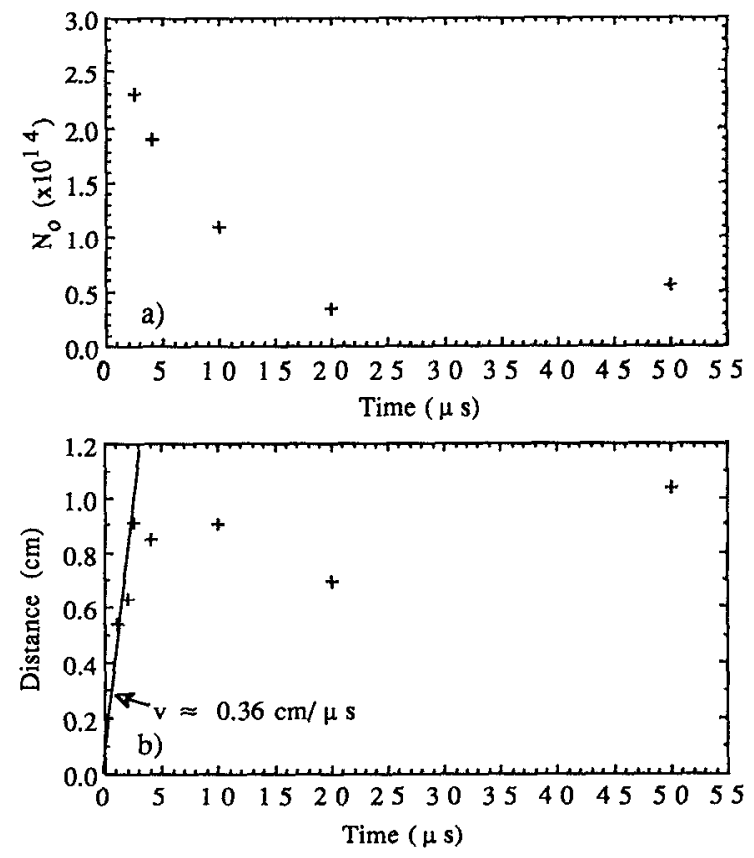

FIG. 14. Time scan in 1 Torr argon gas interpretation: (a) the total number of aluminum neutral atoms $N_{0}$ vs time, and (b) the expansion of the $1 \times 10^{14}$ $\mathrm{cm}^{-2}$ equicontour line vs time.

sity of $1 \times 10^{10} \mathrm{~cm}^{-3}$ and a maximum kinetic temperature of $2 \mathrm{eV}$, thus, the Stark broadening introduced into the aluminum neutral line density calculations due to the rf plasma is minimal. Therefore, the calculations shown above assuming no Stark broadening were used here for the plasma case as well.

The first point in Fig. 15 is that the plume shapes look very similar to the nonplasma case. Second, note the separation of the expanding plume and the residual plume (in contact with the target) at $20.0 \mu$ s in Fig. 15(1) and only the expanding plume at $50.0 \mu \mathrm{s}$ in Fig. 15(n). Third, the number of aluminum atoms in the plume was slightly less for the if plasma case than for the nonplasma case, as shown in Fig. 16(a). Fourth, the $1 \times 10^{14} \mathrm{~cm}^{-2}$ equicontour line appears to expand at about the same rate, yet it was farther away from the target in the rf plasma case than in the gas case, as shown in Fig. 16(b).

At this time it is unknown whether the observed differences are: (1) just shot-to-shot statistical variations of the ablation plumes; (2) caused by the of electric field directly affecting the ablated aluminum ions at very early times $(<0.1 \mu \mathrm{s})$; or (3) caused by collisions between the ablation plume and the energetic electrons and argon ions during the entire expansion time. For example, energetic collisions of the argon plasma with the ablation plume could cause either ionization or condensation of the aluminum atoms, thus causing the observed reduction in the number of neutral atoms. Investigation into these differences is ongoing.

Interferograms were also taken in 35 Torr argon at early times $(<4 \mu \mathrm{s})$ at both near-resonance $(\Delta \lambda=+0.004 \mathrm{~nm})$ and off-resonance $(\Delta \lambda=+0.503 \mathrm{~nm})$ wavelengths. Nonresonant fringe shift was observed at 0.22 and $0.52 \mu \mathrm{s}$, corresponding 
to the lower density of particles inside the laser ablation shock wavc. A point-by-point subtraction of the offresonance fringe shift from the near-resonance fringe shift allows the resonant line density to be determined. Although the Lorentz broadening mechanisms become comparable to the natural broadening as the pressure is increased to 35 Torr, this produces a negligible change in the integral $W(-u+i \alpha)$ for the parameters of this work. From the aforementioned interferograms, the initial expansion velocity of the $1 \times 10^{14}$ $\mathrm{cm}^{-2}$ equiline-density contour was measured to be $\sim 0.08$ $\mathrm{cm} / \mu \mathrm{s}$.
Figure 17 summarizes the total number of aluminum neutral atoms in the ground state measured for all the conditions investigated: vacuum, vacuum deposition, $14 \mathrm{mTorr}, 52$ mTorr, 210 mTorr, 1 Torr, 1 Torr rf plasma, and 35 Torr. The values of $N_{0}$ range from $2.5 \times 10^{12}$ to $3.2 \times 10^{14}$. Since many of the earlier $N_{0}$ in each time scan are $>1 \times 10^{14}$ and since $3 \times 10^{14}$ was the estimated maximum number of $\mathrm{Al}$ neutrals in the laser ablation deposition discussion above, $N_{0} \approx 3 \times 10^{14}$ is a good estimate of the number of aluminum ground-state neutral atoms that the $\mathrm{KrF}$ laser was ablating from the aluminum target, for the experimental parameters in

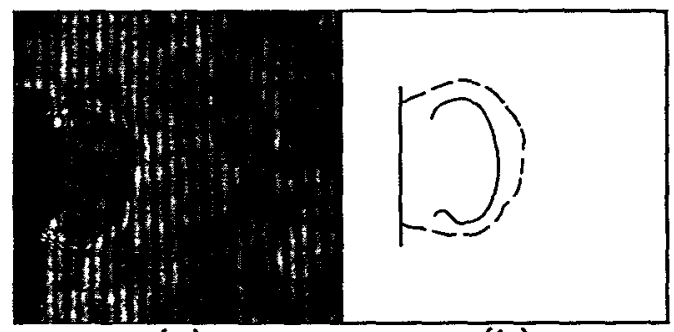

(a)

(b)

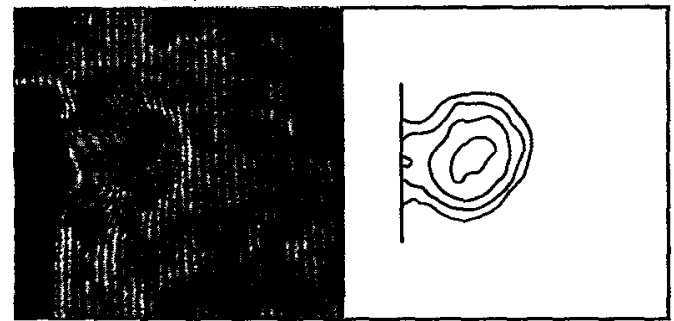

(c)

(d)

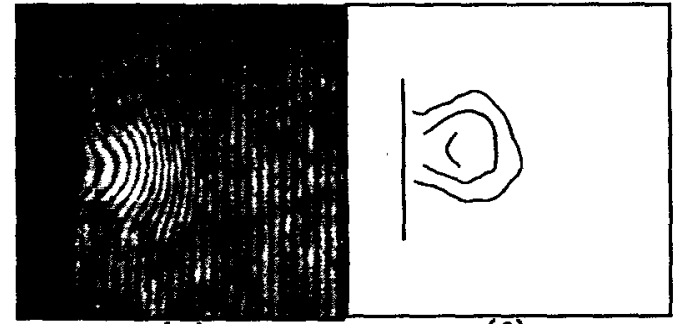

(e)

(f)

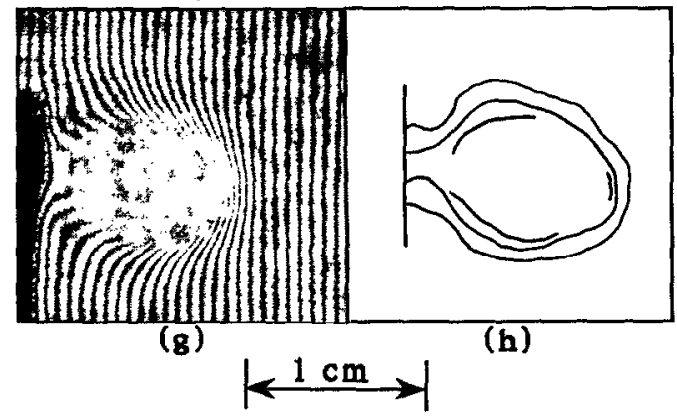

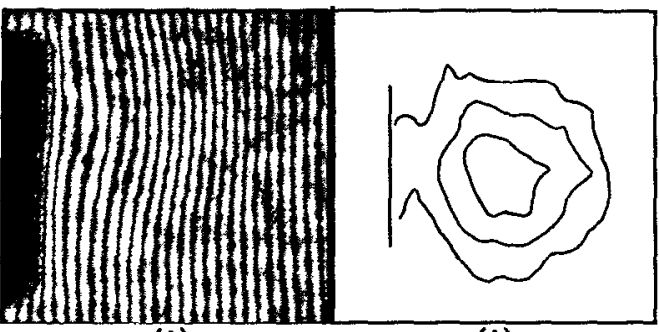

(i)

(j)

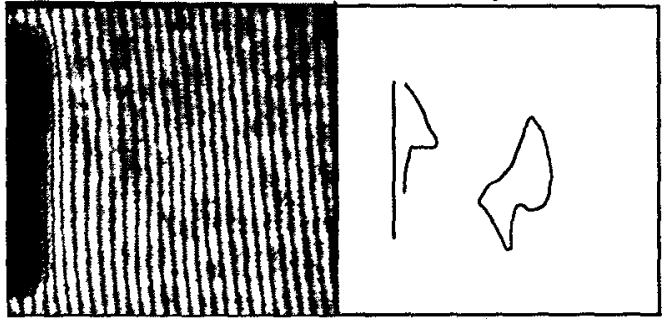

(k)

(1)

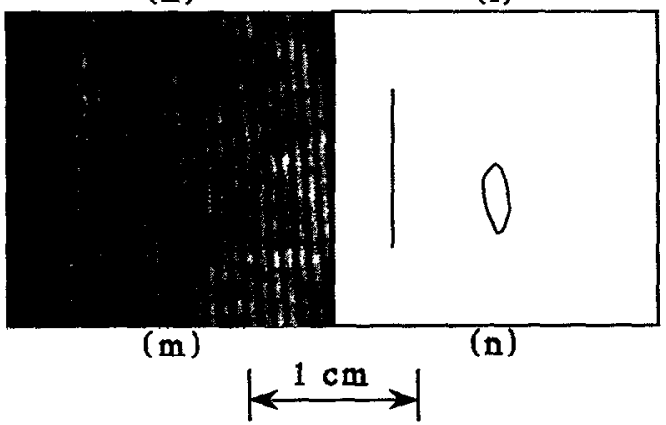

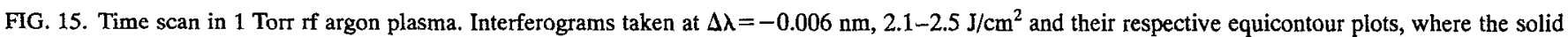

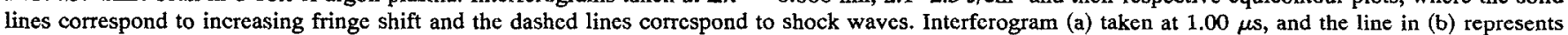

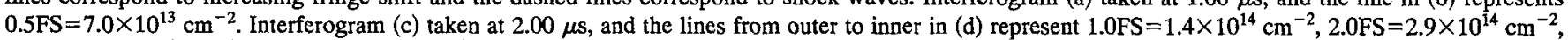

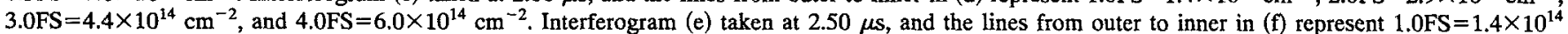

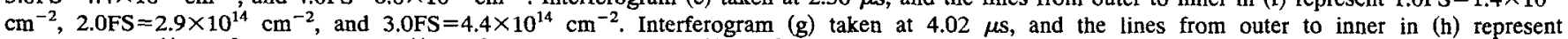

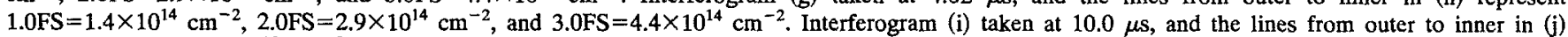

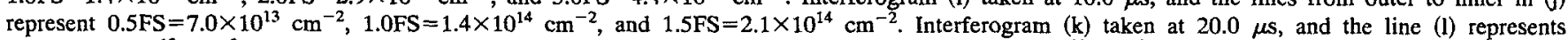
$0.5 \mathrm{FS}=7.0 \times 10^{13} \mathrm{~cm}^{-2}$. Interferogram $(\mathrm{m})$ taken at $50.0 \mu \mathrm{s}$, and the line in (n) represents $0.5 \mathrm{FS}=7.0 \times 10^{13} \mathrm{~cm}^{-2}$. 

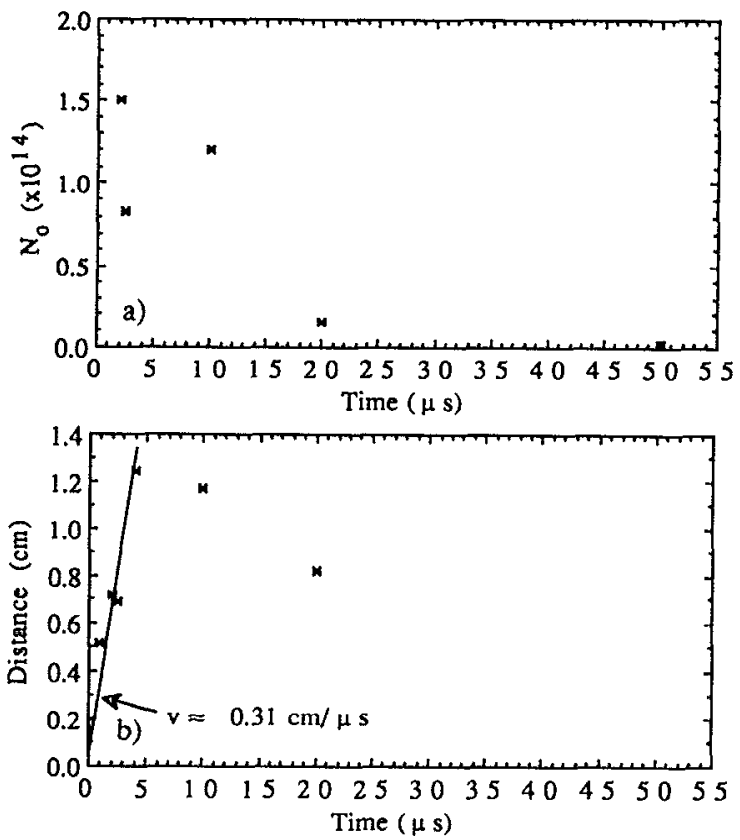

FIG. 16. Time scan in 1 Torr if argon plasma interpretation: (a) the total number of aluminum neutral atoms $N_{0}$ vs time, and (b) the expansion of the $1 \times 10^{14} \mathrm{~cm}^{-2}$ equicontour line vs time.

this work. Again, this is probably a somewhat low estimate since the interferograms represent only aluminum neutral atoms in the ground state.

Figure 18 compiles the approximate expansion velocities of the ablation plumes versus pressure. The vacuum case was $0.2 \mathrm{~m}$ Torr air, but the others were with an argon background. For $210 \mathrm{~m}$ Torr and less, the expansion velocity is in the range $1.1-1.4 \mathrm{~cm} / \mu \mathrm{s}$, while for higher background pressures of argon the expansion velocity decreases to $\approx 0.3 \mathrm{~cm} / \mu \mathrm{s}$ at 1 Torr and then to $\approx 0.08 \mathrm{~cm} / \mu \mathrm{s}$ at 35 Torr.

\section{ACKNOWLEDGMENTS}

This research is supported by National Science Foundation Grant No. CTS-9108971. R.A.L. had a Magnetic Fusion Energy Technology fellowship which was administered for the U.S. Department of Energy by Oak Ridge Associated Universities. J.S.L. has a National Science Foundation Graduate Fellowship. The authors acknowledge D. Steel and Y. Y. Lau for help with the theory, J. Holloway for help with the numerical calculations, and the technical assistance of $A$. Allen, A. Edson, D. Love, R. Schmitz, and O. Valree.

\section{APPENDIX}

To account for the averaging over the Gaussian laser bandwidth of Measures's Voigt profile for smaller $\Delta \lambda$, to account for selective absorption, and to account for the interference effects induced at the holographic plate by a finite bandwidth light source, the temporal-coherence discussions of Goodman for interferometry are utilized in this work. ${ }^{49}$
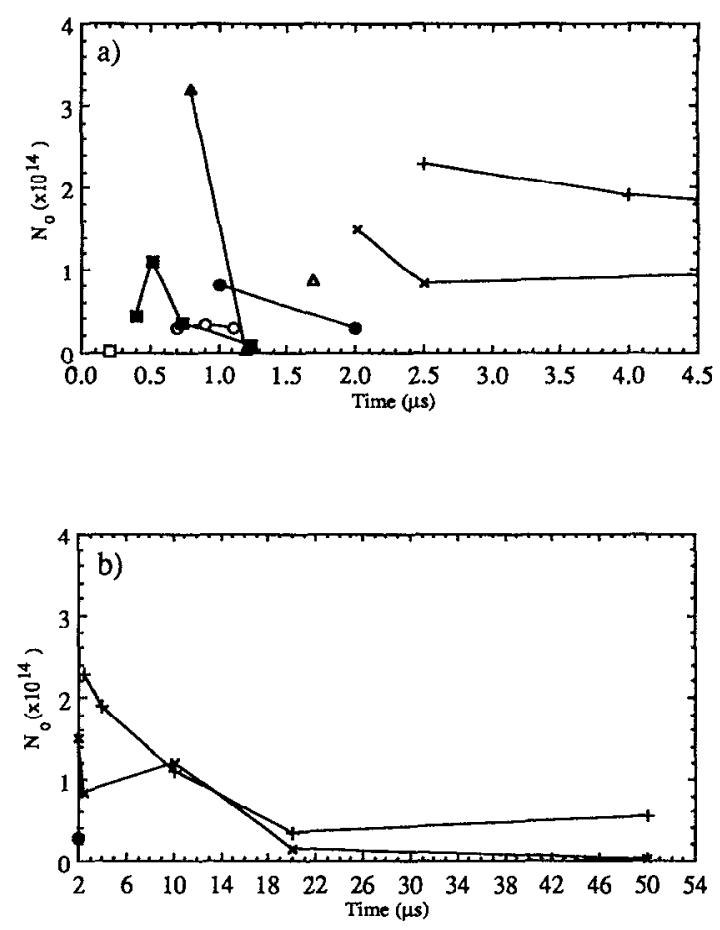

FIG. 17. Interpretation of all time scans in argon at (a) earlier times and (b) later times; the total number of aluminum neutral atoms $N_{0}$ vs time for: (O) vacuum; $(\Delta)$ deposition in vacuum; $(\bullet) 14 \mathrm{mTorr}$ ( $(\Delta) 52 \mathrm{mTorr} ;(\mathbb{D}) 210$ mTorr; (+) 1 Torr gas; (×) 1 Torr rf plasma; and ( $\square$ ) 35 Torr.

Here the laser is simply modeled as producing one photon at a time with a random frequency $\omega$, which has a Gaussian probability distribution about a center frequency $\bar{\omega}$. It is then sufficient to time average the random frequency of the laser. By convention this is written

$$
\begin{aligned}
\Gamma(\tau) & =\left\langle\mathbf{E}(t+\tau) \mathbf{E}^{*}(t)\right\rangle \\
& =\lim _{T \rightarrow \infty} \frac{1}{T} \int_{-T / 2}^{+T / 2} \mathbf{E}(t+\tau) \mathbf{E}^{*}(t) d t,
\end{aligned}
$$

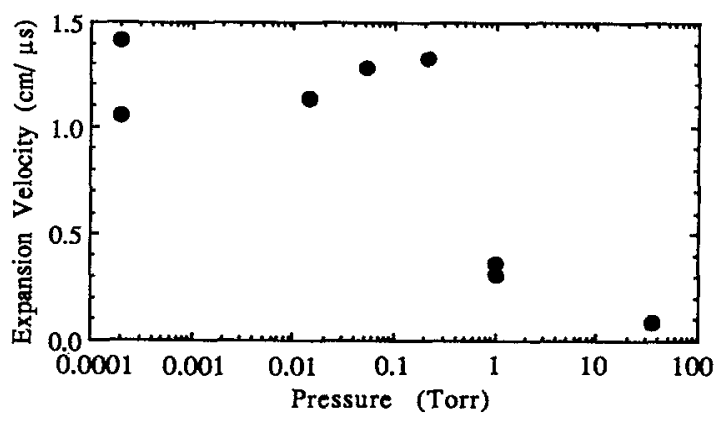

FIG. 18. Interpretation of all time scans in argon; the expansion of the $1 \times 10^{14} \mathrm{~cm}^{-2}$ equicontour line vs pressure. 
where $\Gamma(\tau)$ is the complex autocorrelation function, $\mathbf{E}(t)$ is the complex electric field of the laser, and \langle\rangle denotes a time average.

The electric field of the laser is assumed to be ergodic, thus the Wiener-Khinchin theorem can be used, which transforms $\Gamma(\tau)$ from $t$ space to $\omega$ space. Using a Gaussian frequency distribution, the time average of the electric fields on the holographic plate is then

$\Gamma(\tau)=\frac{2 S^{2} \sqrt{\ln 2}}{\sigma \sqrt{\pi}} \int_{-\infty}^{\infty} \exp \left[-4 \ln 2\left(\frac{\omega-\varpi}{\sigma}\right)^{2}-i \omega \tau\right] d \omega$,

where $\sigma$ is the FWHM of the frequency of the laser intensity. Note that Eq. (A2) only includes temporal coherence (two beams delayed in time) and not spatial coherence (two beams shifted in space). For this work it is assumed that the spatial coherence of the laser is sufficient so that the relevant mathematics may be neglected.

In holographic interferometry, the laser beam is split into two parts, the object beam $O$ and the reference beam $R$. The object beam is passed through the transparent test section, and the two beams are recombined onto a holographic plate. The intensity of light as a function of position on the holographic plate is written as ${ }^{39}$

$$
I=\left|\mathbf{E}_{O}+\mathbf{E}_{R}\right|^{2}=\left|\mathbf{E}_{R}\right|^{2}+\left|\mathbf{E}_{O}\right|^{2}+\mathbf{E}_{O}^{*} \mathbf{E}_{R}+\mathbf{E}_{O} \mathbf{E}_{R}^{*} .
$$

During reconstruction these wave fronts are reproduced and the last term in Eq. (A3) represents the reconstructed test object. To form an interference pattern the initial laser exposing the holographic plate is pulsed twice, once without the test object (1) and once with the test object (2). The intensity of light as a function of position on the film of the reconstruction camera is then

$$
I_{c}=\left|\theta_{0} \mathbf{E}_{C}\left(\mathbf{E}_{O 1} \mathbf{E}_{R 1}^{*}+\mathbf{E}_{O 2} \mathbf{E}_{R 2}^{*}\right)\right|^{2},
$$

where $\theta_{0}$ is the transmission constant for the first-order diffractions of the holographic plate and is dependent on the photographic developing process, and $\mathbf{E}_{c}$ is the electric field of the reconstruction laser beam. To interpret the reconstruction photograph, Eq. (3) is inserted into the term $\mathbf{E}_{O_{2}}$ of Eq. (A4) and the phase shift information $\Delta \phi(x, y)$ is displayed as a black and white pattern on the film.

To account for the finite laser bandwidth, Eq. (A4) is time averaged to give

$$
\left\langle I_{c}\right\rangle=\left|\theta_{0} \mathbf{E}_{\mathrm{c}}\left(\boldsymbol{\Gamma}_{O R 1}+\boldsymbol{\Gamma}_{O R 2}\right)\right|^{2} .
$$

The bandwidth of the reconstruction laser is assumed to be infinitesimal.

Recalling the notation for the electric fields given in Eqs. (1) and (2), the two laser beams for both laser pulses can be written

$$
\Phi_{O 1}=\frac{\omega z}{c}+\omega \tau_{O}-i K_{O}
$$

$$
\begin{aligned}
& \Phi_{R 1}=\frac{\omega z}{c}+\omega \tau_{R}-i K_{R}, \\
& \Phi_{O 2}=\frac{\omega z}{c}+\omega \tau_{O}-i K_{O}-i A \overline{N_{i} L} W(-u+i \alpha),
\end{aligned}
$$

and

$$
\Phi_{R 2}=\frac{\omega z}{c}+\omega \tau_{R}-i K_{R}+\omega y \delta
$$

where $K_{O}$ and $K_{R}$ represent any absorption incurred outside the test section by the object and reference beams, respectively, and $\tau_{O}$ and $\tau_{R}$ refer to the travel time of the object and reference beams, respectively, as they travel between the incident side of the beam splitter and the holographic plate.

The Doppler-broadened test section $\left[-i A \overline{N_{i} L} W(-u\right.$ $+i \alpha)]$ is now included in $\Phi_{O 2}$ and the reference fringes caused by turning a mirror in the reference beam between laser pulses $\omega y \delta$ are accounted for in $\Phi_{R 2}$. This notation for reference fringes means that the black and white stripes are in the $\hat{x}$ direction with the variation in the $\hat{y}$ direction, whose spacing is determined by the constant $\delta$.

Now inserting Eqs. (A6) and (A7) into Eq. (A2), where $\omega \tau$ is equivalent to $\left(\Phi_{O}-\Phi_{R}^{*}\right)$, one obtains

$$
\begin{aligned}
\Gamma_{O R 1}= & S_{O} S_{R} \exp \left(-K_{O}-K_{R}-\frac{\sigma^{2}\left(\tau_{O}-\tau_{R}\right)^{2}}{16 \ln 2}\right. \\
& \left.-i \bar{\omega}\left(\tau_{O}-\tau_{R}\right)\right)
\end{aligned}
$$

where the $\sigma^{2}\left(\tau_{O}-\tau_{R}\right)^{2} /(16 \ln 2)$ term in Eq. (A10) represents temporal coherence. If the timc-travcl differcnce bctween the object and reference beams becomes much larger than the coherence time of the laser, $(2 \pi / \sigma)$, then $\boldsymbol{\Gamma}_{O R 1} \rightarrow 0$ and no fringes are observed.

Equations (A8), (A9), and (A2) combine to give

$$
\Gamma_{O R 2}=\frac{2 S_{0} S_{R} \beta \sqrt{\ln 2}}{\sigma \sqrt{\pi}} \int_{-\infty}^{\infty} d \epsilon \exp [g(\epsilon)]
$$

where

$$
\begin{aligned}
g(\epsilon)= & -4 \ln 2\left(\frac{\beta}{\sigma}\right)^{2} \epsilon^{2}-K_{O}-K_{R}-i \bar{\omega}\left(\tau_{O}-\tau_{R}-\delta y\right) \\
& -A \overline{N_{i} L} W(\Theta),
\end{aligned}
$$

and where $\omega^{\prime}=\omega-\bar{\omega}, \Delta=\left(\bar{\omega}-\omega_{0}\right) / \beta, \epsilon=\omega^{\prime} / \beta, u=\Delta+\epsilon$, and $\theta=-\Delta-\epsilon+i \alpha . \Gamma_{O R 2}$ is not analytically solvable because of the presence of $W(z)$ in the exponential, so its solution is approximated in this work using the saddle-point theorem, ${ }^{50}$ 


$$
\begin{aligned}
& \int_{-\infty}^{\infty} d x \exp [f(x)] \\
& \cong \int_{-\infty}^{\infty} d x \exp \left(f\left(x_{s}\right)+\frac{1}{2} f^{\prime \prime}\left(x_{s}\right)\left(x-x_{s}\right)^{2}\right) \\
& \cong \eta \exp \left[f\left(x_{s}\right)\right]\left(\frac{1}{f^{\prime \prime}\left(x_{s}\right)}\right)^{1 / 2},
\end{aligned}
$$

where $\eta$ is a constant and $x_{s}$ is a solution of $f^{\prime}\left(x_{s}\right) \equiv 0$.

The $\omega^{\prime}\left(\tau_{O}-\tau_{R}-\delta y\right)$ term has been dropped to make the integration possible, thereby assuming $\omega^{\prime} \ll \bar{\omega}$ for the values of $\omega^{\prime}$ where the $4 \ln 2\left(\omega^{\prime} / \sigma\right)^{2}$ term does not dominate in the exponential of Eq. (A11). The condition $g^{\prime}\left(\epsilon_{s}\right)=0$ was solved for the complex value $\epsilon_{\mathrm{s}}$ using Newton's Method in Mathematica on the Macintosh computer. Two roots were found. One of the roots changed continuously as $\Delta$ and $N L$ were varied, while the other root was discontinuous for the same parameter regions. Therefore, the first root was used in the solutions that follow.

The solution to Eq. (A5) can now be written

$$
\left\langle I_{c}\right\rangle=M \exp (-N)\left[1+P^{2}+2 P \cos (Q)\right],
$$

where

$$
\begin{aligned}
& M=\theta_{0}^{2}\left|\mathbf{E}_{C}\right|^{2} S_{O}^{2} S_{R}^{2}, \\
& N=2 K_{O}+2 K_{R}+\frac{\sigma^{2}\left(\tau_{O}-\tau_{R}\right)^{2}}{8 \ln 2}, \\
& P=\left(\frac{g^{\prime \prime}(0)}{g^{\prime \prime}\left(\epsilon_{\mathrm{s}}\right)}\right)^{1 / 2} \exp \left[R\left(\epsilon_{\mathrm{s}}\right)\right], \\
& Q=\bar{\omega} y \delta+Y\left(\epsilon_{\mathrm{s}}\right),
\end{aligned}
$$

and where

$$
R\left(\epsilon_{s}\right)=\operatorname{Re}\left[-4 \ln 2\left(\frac{\beta}{\sigma}\right)^{2} \epsilon_{s}^{2}-A \overline{N_{i} L} W\left(-\Delta-\epsilon_{s}+i \alpha\right)\right],
$$

$Y\left(\epsilon_{\mathrm{s}}\right)=\operatorname{Im}\left[-4 \ln 2\left(\frac{\beta}{\sigma}\right)^{2} \epsilon_{s}^{2}-A \overline{N_{i} L} W\left(-\Delta-\epsilon_{\mathrm{s}}+i \alpha\right)\right]$.

Since the reference fringes $\varpi y \delta$ are manually removed when the reconstructed interferograms are analyzed, that term may be dropped here and thus the resonant fringe shift can be interpreted as

$$
\begin{aligned}
\left(\frac{\Delta s}{s}\right)= & \frac{Q}{2 \pi}=\frac{1}{2 \pi} \operatorname{Im}\left[-4 \ln 2\left(\frac{\beta}{\sigma}\right)^{2} \epsilon_{s}^{2}\right. \\
& \left.-A \overline{N_{i} L} W\left(-\Delta-\epsilon_{\mathrm{s}}+i \alpha\right)\right] .
\end{aligned}
$$

Note that as the diagnostic laser bandwidth becomes infinitesimal in Eq. (A11) and thus $\epsilon_{s} \rightarrow 0$ in the solution to $g^{\prime}\left(\boldsymbol{\epsilon}_{\mathbf{s}}\right)=0$, Measures's answer is obtained as in Eq. (5).
${ }^{1}$ R. Srinivasan, J. Appl. Phys. 73, 2743 (1993); A. Gupta, B. Braren, K. G. Casey, B. W. Hussey, and R. Kelly, Appl. Phys. Lett. 59, 1302 (1991).

${ }^{2}$ H.-J. Siebeneck, D. K. Koopman, and J. A. Cobble, Rev. Sci. Instrum. 48, 997 (1977).

${ }^{3}$ G. Jellison and C. R. Parsons, Phys. Fluids 24, 1787 (1981).

${ }^{4}$ A. N. Mostovych, B. H. Ripin, and J. A. Stamper, Rev. Sci. Instrum. 59, 1497 (1988).

${ }^{5}$ R. M. Gilgenbach and P. L. G. Ventzek, Appl. Phys. Lett. 58, 1597 (1991).

${ }^{6}$ P. L. G. Ventzek, R. M. Gilgenbach, C. H. Ching, and R. A. Lindley, J. Appl. Phys. 72, 1696 (1992).

${ }^{7}$ D. B. Geohegan, Appl. Phys. Lett. 60, 2732 (1992); 62, 1463 (1993).

${ }^{8}$ M. A. Cappelli, P. H. Paul, and R. K. Hanson, Appl. Phys. Lett. 56, 1715 (1990).

${ }^{9}$ A. D. Sappey and T. K. Gamblc, J. Appl. Phys. 72, 5095 (1992).

${ }^{10}$ P. L. G. Ventzek, R. M. Gilgenbach, J. A. Sell, and D. M. Heffelfinger, J. Appl. Phys. 68, 965 (1990).

${ }^{11}$ D. K. Koopman, H.-J. Siebeneck, and G. Jellison, Phys. Fluids 22, 526 (1979).

${ }^{12}$ R. E. Walkup, J. M. Jasinski, and R. W. Dreyfus, Appl. Phys. Lett. 48, 1690 (1986).

${ }^{13}$ G. V. Dreiden, A. N. Zaidel', G. V. Ostrovskaya, Yu. I. Ostrovskii, N. A. Pohedonostseva, L. V. Tanin, V. N. Filippov, and E. N. Shedova, Sov. J. Plasma Phys. 1, 256 (1975).

${ }^{14}$ R. A. Lindley, R. M. Gilgenbach, and C. H. Ching, Appl. Phys. Lett. 63, 888 (1993).

${ }^{15}$ J. T. Dickinson, S. C. Langford, L. C. Jensen, P. A. Eschbach, L. R. Pederson, and D. R. Baer, J. Appl. Phys. 15, 1831 (1990).

${ }^{16} \mathrm{~J}$. S. Meachum, Ph.D. thesis, University of Michigan, Ann Arbor, MI, 1988.

${ }^{17}$ R. J. von Guttfield and R. W. Dreyfus, Appl. Phys. Lett. 54, 1212 (1989)

${ }^{18}$ D. Feldman, J. Kutzner, J. Laukemper, S. MacRobert, and K. H. Welge, Appl. Phys. B 44, 81 (1987); H. Cronberg, M. Reichling, E. Broberg, H. B. Nielsen, E. Matthias, and N. Tolk, ibid. 52, 155 (1991).

${ }^{19}$ C. L. Enloe, R. M. Gilgenbach, and J. S. Meachum, Rev. Sci. Instrum. 58, 1597 (1987).

${ }^{20}$ P. L. G. Ventzek, R. M. Gilgenbach, D. M. Heffelfinger, and J. A. Sell, J. Appl. Phys. 70, 587 (1991).

${ }^{21}$ J. A. Sell, D. M. Heffelfinger, P. L. G. Ventzek, and R. M. Gilgenbach, Appl. Phys. Lett. 55, 2435 (1989); J. Appl. Phys. 69, 1330 (1991).

${ }^{22}$ N. H. Cheung, Q. Y. Ying, J. P. Zheng, and H. S. Kwok, J. Appl. Phys. 69 , 6349 (1991).

${ }^{23}$ D. B. Geohegan and D. N. Mashburn, Appl. Phys. Lett. 55, 2345 (1989).

${ }^{24}$ M. L. Brake, J. Meachum, R. M. Gilgenbach, and W. Thornhill, IEEE Trans. Plasma Sci. PS-15 (1987).

${ }^{25} \mathrm{~K}$. Murakami, in Laser Ablation of Electron Materials, edited by E. Fogarassy and S. Lazare (North-Holland, New York, 1992), Vol. 4, p. 125.

${ }^{26}$ R. W. Dreyfus, R. Kelly, and R. E. Walkup, Appl. Phys. Lett. 49, 1478 (1986); R. W. Dreyfus, J. Appl. Phys. 69, 1721 (1991).

${ }^{27}$ H. Wang, A. P. Saltzberg, and B. R. Weiner, Appl. Phys. Lett. 59, 935 (1991).

${ }^{28}$ A. D. Sappey and T. K. Gamble, Appl. Phys. B 53, 353 (1991).

${ }^{29}$ J. S. Bakos, P. N. Ignacz, and J. Szigeti, Appl. Phys. Lett. 51, 734 (1987).

${ }^{30}$ T. P. Duffey, T. G. McNeela, J. Mazumder, and A. L. Shawlow, Appl. Phys. Lett. 63, 2339 (1993).

${ }^{31}$ A. D. Sappey, T. K. Gamble, and D. K. Zerkle, Appl. Phys. Lett. 62, 564 (1993).

${ }^{32}$ L. Wang, K. W. D. Ledingham, C. J. McLean, and R. P. Singhal, Appl. Phys. B 54, 71 (1992).

${ }^{33}$ R. Kelly and J. E. Rothenburg, Nucl. Instrum. Methods Phys. Res. B 7/8, 755 (1985).

${ }^{34}$ R. Srinivasan and B. Braren, J. Polym. Sci. 22, 2601 (1984).

${ }^{35}$ R. J. Contolini and J. Alspector, J. Appl. Phys. 65, 1759 (1989).

${ }^{36}$ H. R. Griem, Plasma Spectroscopy (McGraw-Hill, New York, 1964).

${ }^{37} \mathrm{D}$. Bershader, in Modern Optical Methods in Gas Dynamic Research, edited by D. S. Dosanjh (Plenum, New York, 1971).

${ }^{38}$ R. M. Measures, Appl. Opt. 9, 737 (1970).

${ }^{39}$ C. M. Vest, Holographic Interferometry (Wiley, New York, 1979).

${ }^{40}$ A. Miotello, R. Kelly, B. Braren, and C. E. Otis, Appl. Phys. Lett. 61, 2784 (1992).

${ }^{41}$ I. NoorBatcha, R. R. Lucchese, and Y. Zeiri, J. Chem. Phys. 86, 5816 (1987).

${ }^{42}$ R. Kelly and R. W. Dreyfus, Nucl. Instrum. Mcthods Phys. Res. B 32, 341 (1988). 
${ }^{43}$ N. G. Utterback, S. P. Tang, and J. F. Friichtenicht, Phys. Fluids 19, 900 (1976).

${ }^{44}$ G. J. Tallents, Laser and Particle Beams 1, 171 (1983).

${ }^{45}$ J. C. S. Kools, T. S. Baller, S. T. De Zwart, and J. Dieleman, J. Appl. Phys. 71, 4547 (1992).

${ }^{46}$ K. L. Saenger, J. Appl. Phys. 70, 5029 (1991).
${ }^{47}$ G. K. Batchelor, An Introduction to Fluid Dynamics (Cambridge University Press, London, 1967), Plates 20 and 21.

${ }^{48} \mathrm{R}$. Kelly (private communication).

${ }^{49} \mathrm{~J}$. W. Goodman, Statistical Optics (Wiley-Interscience, New York, 1985).

${ }^{50} \mathrm{G}$. Arfken, Mathematical Methods for Physicists, 3rd ed. (Academic/ Harcourt Brace Jovanovich, San Diego, CA, 1985). 